\title{
The SEDs and host galaxies of the dustiest GRB afterglows ${ }^{\star}$
}

\author{
T. Krühler ${ }^{1,2,3}$, J. Greiner ${ }^{1}$, P. Schady ${ }^{1}$, S. Savaglio ${ }^{1}$, P. M. J. Afonso ${ }^{1,4}$, C. Clemens ${ }^{1}$, J. Elliott ${ }^{1}$, R. Filgas ${ }^{1}$, D. Gruber ${ }^{1}$,
} D. A. Kann ${ }^{5}$, S. Klose ${ }^{5}$, A. Küpcü-Yoldaş ${ }^{6}$, S. McBreen ${ }^{7}$, F. Olivares ${ }^{1}$, D. Pierini ${ }^{\star \star}$, A. Rau ${ }^{1}$, A. Rossi ${ }^{5}$, M. Nardini ${ }^{1,8}$, A. Nicuesa Guelbenzu ${ }^{5}$, V. Sudilovsky ${ }^{1}$, and A. C. Updike ${ }^{9,10}$

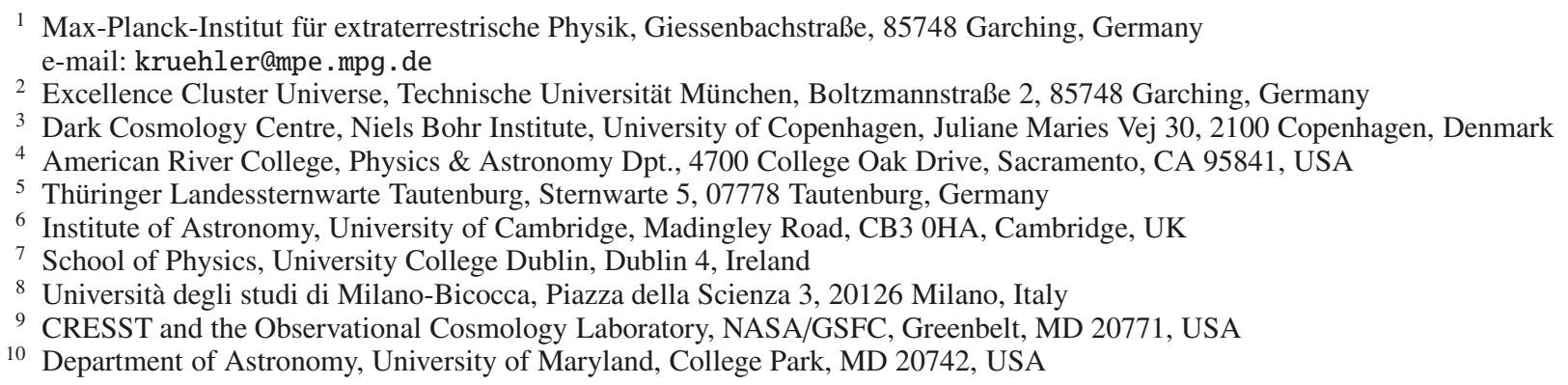

Received 7 June 2011 / Accepted 9 August 2011

\begin{abstract}
Context. The afterglows and host galaxies of long gamma-ray bursts (GRBs) offer unique opportunities to study star-forming galaxies in the high- $z$ Universe. Until recently, however, the information inferred from GRB follow-up observations was mostly limited to optically bright afterglows, biasing all demographic studies against sight-lines that contain large amounts of dust.

Aims. Here we present afterglow and host observations for a sample of bursts that are exemplary of previously missed ones because of high visual extinction $\left(A_{\mathrm{V}}^{\mathrm{GRB}} \gtrsim 1 \mathrm{mag}\right.$ ) along the sight-line. This facilitates an investigation of the properties, geometry, and location of the absorbing dust of these poorly-explored host galaxies, and a comparison to hosts from optically-selected samples.

Methods. This work is based on GROND optical/NIR and Swift/XRT X-ray observations of the afterglows, and multi-color imaging for eight GRB hosts. The afterglow and galaxy spectral energy distributions yield detailed insight into physical properties such as the dust and metal content along the GRB sight-line and galaxy-integrated characteristics such as the host's stellar mass, luminosity, color-excess, and star-formation rate.

Results. For the eight afterglows considered in this study, we report for the first time the redshift of GRB $081109(z=0.9787 \pm 0.0005)$, and the visual extinction towards GRBs $081109\left(A_{\mathrm{V}}^{\mathrm{GRB}}=3.4_{-0.3}^{+0.4} \mathrm{mag}\right)$ and $100621 \mathrm{~A}\left(A_{\mathrm{V}}^{\mathrm{GRB}}=3.8 \pm 0.2 \mathrm{mag}\right)$, which are among the largest ever derived for GRB afterglows. Combined with non-extinguished GRBs, there is a strong anti-correlation between the afterglow's metal-to-dust ratio and visual extinction. The hosts of the dustiest afterglows are diverse in their properties, but on average redder $\left(\left\langle(R-K)_{\mathrm{AB}}\right\rangle \sim 1.6 \mathrm{mag}\right)$, more luminous $\left(\langle L\rangle \sim 0.9 L^{*}\right)$, and massive $\left(\left\langle\log M_{*}\left[M_{\odot}\right]\right\rangle \sim 9.8\right)$ than the hosts of optically-bright events. Hence, we probe a different galaxy population, suggesting that previous host samples miss most of the massive and metal-rich members. This also indicates that the dust along the sight-line is often related to host properties, and thus probably located in the diffuse ISM or interstellar clouds and not in the immediate GRB environment. Some of the hosts in our sample, are blue, young, or of low stellar mass illustrating that even apparently non-extinguished galaxies possess very dusty sight-lines owing to a patchy dust distribution.

Conclusions. The afterglows and host galaxies of the dustiest GRBs provide evidence of a complex dust geometry in star-forming galaxies. In addition, they establish a population of luminous, massive, and correspondingly chemically evolved GRB hosts. This suggests that GRBs trace the global star-formation rate better than studies based on optically selected host samples indicate, and that the previously claimed deficiency of high-mass hosts was at least partially a selection effect.
\end{abstract}

Key words. gamma-ray burst: general - dust, extinction - galaxies: star formation

\section{Introduction}

Long gamma-ray bursts (GRBs, see e.g., Zhang 2007; Gehrels et al. 2009, for reviews) are linked to core-collapse supernovae, hence star formation via the death of massive stars (e.g., Galama et al. 1998; Hjorth et al. 2003). At high redshifts,

* Based on observations made with GROND at the MPI/ESO $2.2 \mathrm{~m}$ telescope and with telescopes at the European Southern Observatory at LaSilla/Paranal, Chile under program 086.A-0533 and obtained from the ESO/ST-ECF Science Archive Facility from programs 177.A-0591 and 078.D-0416.

$\star \star$ Visiting Astronomer at MPE. where a significant fraction of star formation is thought to be dust-obscured (e.g., Adelberger \& Steidel 2000; Chapman et al. 2005), GRBs and their host galaxies provide independent means of improving the understanding and preforming a full census of star formation in the early Universe (e.g., Blain et al. 2000; Ramirez-Ruiz et al. 2002; Berger et al. 2003): GRBs, having luminous emission in a simple power-law spectrum provide the ideal background light to illuminate dust-enshrouded starforming regions that would otherwise remain unexplored, while at the same time pinpointing their host galaxies.

However, the extent to which GRB hosts provide an unbiased picture of the formation of high-mass stars, and whether they 
preferentially occur in low-metallicity environments remains a much debated issue (e.g., Le Floc'h et al. 2003; Fynbo et al. 2003; Tanvir et al. 2004; Fruchter et al. 2006; Kocevski et al. 2009; Campisi et al. 2011). In single progenitor models, metalpoor stars are predicted to be more likely to explode as a GRB (Woosley 1993; MacFadyen \& Woosley 1999), as they would in principle be able to retain a larger amount of angular momentum at the time of stellar collapse because of the lower wind pressures and losses throughout their evolution (e.g., Yoon \& Langer 2005; Mokiem et al. 2007). However, binary progenitor channels might also play an important role in the formation of long GRBs (e.g., Fryer et al. 1999), having somewhat relaxed metallicity constraints relative to single star progenitors (Fryer et al. 2007). Observations of GRB hosts are hence not only important in a cosmological context, but provide relevant clues to the exact nature of GRB progenitors.

A fundamental limit of hitherto available GRB host galaxy samples is the incompleteness which arises from the nondetection of the optical afterglow of a GRB (e.g., Groot et al. 1998; Fynbo et al. 2001). These optically dark bursts could be caused by either high-redshift (e.g., Greiner et al. 2009; Tanvir et al. 2009; Salvaterra et al. 2009; Cucchiara et al. 2011), large column densities of dust (e.g., Klose et al. 2000, 2003; Tanvir et al. 2008; Perley et al. 2011b) or an intrinsically fainter optical afterglow than the extrapolation of X-ray data when using synchrotron emission theory, i.e., a decoupled optical/X-ray afterglow light-curve (e.g., Panaitescu et al. 2006; Ghisellini et al. 2009; Nardini et al. 2010). New afterglow samples have become available since the advent of dedicated afterglow follow-up campaigns on medium-to-large aperture telescopes (e.g., Fynbo et al. 2009a; Cenko et al. 2009; Greiner et al. 2011). These new afterglow samples reach completeness levels of $\sim 90 \%$ (Greiner et al. 2011) and helped ascertain the origin of dark bursts: around three quarters of dark bursts are the result of a dusty afterglow line of sight (e.g., Perley et al. 2009; Greiner et al. 2011). Accurate positions from afterglow observations are necessary to be able to unambiguously associate galaxies with GRBs. The lack of optical/NIR afterglows for dark GRBs therefore creates a systematic bias against the host galaxies of dusty GRBs in previous studies.

The sample of identified host galaxies is therefore not as comprehensive as the most recent afterglow samples. It is instead largely based on optically bright afterglows and consists mainly of young and vigorously star-forming galaxies with sub- $L^{*}$ luminosities and masses around $10^{9} M_{\odot}$ (e.g., Bloom et al. 1998; Le Floc'h et al. 2003; Christensen et al. 2004; Fruchter et al. 2006; Michałowski et al. 2008; Savaglio et al. 2009, referenced as SGL09, hereafter). However, it remains unclear whether this is a physical consequence of GRBs residing mainly in lowmetallicity environments, or is merely a selection effect: host galaxies of dark GRBs were typically not identified, and hence are under-represented in the available host sample.

Whether the physical characteristics of the hosts of optically dark and bright GRBs are different is also the subject of discussion. Previous sample studies (e.g., Berger et al. 2003; Le Floc'h et al. 2003; Perley et al. 2009) have not revealed strong evidence of a strong diversity. A handful of single dark GRBs have however been found to be hosted by red and dusty galaxies with high metallicities and stellar masses over $10^{11} M_{\odot}$ (e.g., Levan et al. 2006; Berger et al. 2007; Levesque et al. 2010; Hashimoto et al. 2010; Küpcü Yoldaş et al. 2010; Chen et al. 2010). Recently Perley et al. (2010a, 2011a) indicate that the general galaxy population hosting dark bursts is redder and more luminous than those selected via optically bright afterglows.

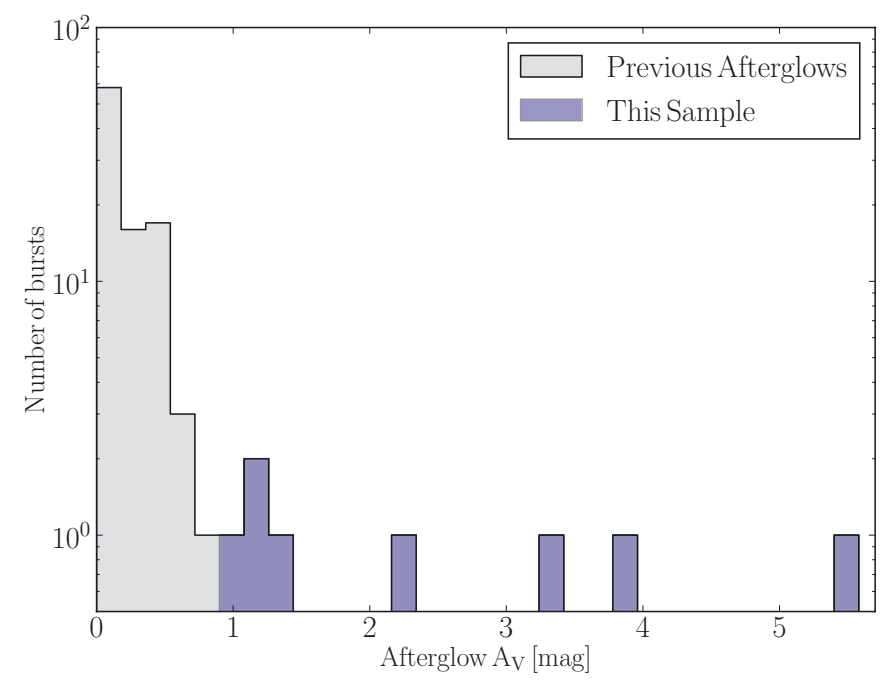

Fig. 1. Histogram of visual extinction values from previous afterglows and those selected for this study. We note that five of the afterglows in this work (GRBs 070802, 080605, 080607, 080805, 090926B) were already discussed in previous sample studies, but are only included in one of the histograms for clarity.

In this paper, we study the nature of GRB hosts that previously escaped detection because of the dust bias, hence are exemplary of those missing from demographic studies. We avail ourselves of dedicated GRB afterglow campaigns of high completeness to preselect the GRB hosts for this study. These afterglow data do not only provide accurate positions for host identifications, but for the first time allow us to directly select dust-extinguished (and not only optically faint) GRBs using well-sampled broad-band (NIR to X-ray) afterglow observations.

After selecting afterglows with visual extinctions $A_{\mathrm{V}}^{\mathrm{GRB}}$ exceeding unity, we search for the associated hosts with the Gamma-Ray Optical and Near-infrared Detector (GROND, Greiner et al. 2008), as well as the ESO Very Large and New Technology Telescopes (VLT and NTT, respectively) and the Ultra-Violet Optical Telescope (UVOT, Roming et al. 2005) onboard the Swift satellite (Gehrels et al. 2004).

The acquired data allow us to study the physical properties of the hosts of high- $A_{\mathrm{V}}^{\mathrm{GRB}} \mathrm{GRBs}$ in detail, and to investigate the bias against dust in GRB host samples. As an ultimate consequence, they address the role of GRB host galaxies as tracers of galaxy formation and evolution. Furthermore, they link afterglow diagnostics, i.e., detailed information about a single sight-line, to integrated host characteristics, and in this combination directly probe the nature of dust and its properties in high-redshift, starforming galaxies.

Throughout this work, we adopt the convention that the flux density of the afterglow $F_{v}(v, t)$ can be described as $F_{v}(v, t) \propto$ $v^{-\beta} t^{-\alpha}$, and assume concordance $\left(\Omega_{\mathrm{M}}=0.27, \Omega_{\Lambda}=0.73\right.$, $\left.H_{0}=71 \mathrm{~km} \mathrm{~s}^{-1} \mathrm{Mpc}^{-1}\right) \Lambda \mathrm{CDM}$ cosmology. All errors are given at $1 \sigma$ confidence levels unless indicated otherwise. All magnitudes and colors are given or converted into the $\mathrm{AB}$ system.

\section{Sample selection}

The host galaxy sample presented in this work is based on a direct measurement of large visual extinction along the GRB line of sight $\left(A_{\mathrm{V}}^{\mathrm{GRB}} \gtrsim 1 \mathrm{mag}\right)$ from multi-color (NIR 
to X-ray) afterglow observations. Specifically, eight GRB afterglows (GRBs 070306, 070802, 080605, 080607, 080805, 081109, 090926B, and 100621A) fulfill the selection criterion and define our initial host sample. Our host sample is a direct result of afterglow observations. The selection itself is hence neither limited by galaxy brightness, nor introduces a bias towards luminous galaxies in our sample. Afterglow measurements for the initial selection have been obtained from the literature or by analyzing photometric optical/NIR data from the GROND archive. In the latter case, they are detailed in Sect. 3.1.

Our eight GRBs have a median redshift of $\left\langle z_{A_{V}}\right\rangle=1.5$. This is significantly lower than the published mean of Swift GRBs with measured redshifts $\left(\left\langle z_{\text {Swift }}\right\rangle=1.9\right.$, Fynbo et al. $\left.2009 \mathrm{a}^{1}\right)$, but higher than the one of the previous host sample $\left(\left\langle z_{\text {SGL09 }}\right\rangle=0.96\right.$ from SGL09), which includes a large number of pre-Swift events. Within the selected eight afterglows, four of them display a clear $2175 \AA$ dust feature (GRBs 070802, 080605, 080607, 080805), which is with the exception of GRB 080603A (Guidorzi et al. 2011; Kann et al. 2011) the full sample for which a significant detection of this feature has been reported to date (e.g., Zafar et al. 2011). The $2175 \AA$ feature was indeed undetectable for the rest of the sample because of the combination of a large dust column, and insufficiently deep and rapid follow-up observations in the case of GRBs 070306 and 090926B, or a lack of observational wavelength coverage at $2175 \AA \times(1+z)$ (GRBs 081109, 100621A).

While observationally challenging, the requirement of a dust measurement has several obvious advantages over a selection based on the optical-to-X-ray flux ratio $\left(\beta_{\mathrm{oX}}\right.$, see e.g., Jakobsson et al. 2004; Rol et al. 2005; van der Horst et al. 2009). Most importantly, the selection is the result of a measurement rather than an extrapolation and provides a clean selection of dusty GRBs, that is our afterglows are chosen according to their visual extinction instead of their optical faintness alone. Optically faint events could of course also be at high- $z$ or have different emission mechanisms in the optical and X-ray regime.

We note that our selection is still somewhat modeldependent, particularly we assume that the afterglow emits synchrotron radiation in the optical/NIR and X-ray regime. Despite the lack of conclusive alternatives to the standard synchrotron fireball model, there are still features in well-sampled multicolor light curves which remain hard to explain. These features include for example chromatic ${ }^{2}$ breaks, optical and/or X-ray flares, or plateaus (e.g., Panaitescu et al. 2006; Covino et al. 2008; Evans et al. 2009; Krühler et al. 2009; Oates et al. 2011, also illustrated in Fig. 3). The apparently decoupled optical and $\mathrm{X}$-ray light curve for some bursts results in a strong dependence of $\beta_{\mathrm{oX}}$ on the time of the observation (which in fact is directly observed in some cases, see e.g., Filgas et al. 2011), hence the dark-burst definition depends on observational constraints and not only physical properties.

In addition, the detection of an optical and/or NIR afterglow yields the GRB position to sub-arcsec accuracy, and hence negligible chance-coincidence probabilities for field galaxies, which is particularly important for samples of small size as in this work. For all events, a spectroscopic redshift is available from the literature, or could be obtained via host-galaxy spectroscopy. This enables quantitative studies, such as the comparison between

\footnotetext{
1 Including updates from http://www.mpe.mpg.de/ jcg/grbgen. html and http://www.raunvis.hi.is/ pja/GRBsample.html

2 Chromatic light-curve breaks are breaks in the X-ray regime not associated with contemporaneous breaks in the optical/NIR bands, and vice versa.
}

specific sight-lines and host-integrated properties, as well as an investigation of the relation of the dusty hosts to the host sample of SGL09.

Owing to the inherent difficulties in accurately localizing high- $A_{\mathrm{V}}^{\mathrm{GRB}}$ afterglows, most previous afterglow samples are biased towards small visual extinctions. This is illustrated in Fig. 1, which compares the visual extinction of GRBs selected for this work to previously compiled $A_{\mathrm{V}}^{\mathrm{GRB}}$ values. The latter were taken from Kann et al. (2006, 2010), Schady et al. (2010), Greiner et al. (2011), and Zafar et al. (2011).

\section{Observations}

\subsection{Afterglows}

Optical and near-infrared measurements of the afterglows of GRBs 070306, 070802, 080605, 080607, 080805, and 090926B or results thereof are taken from Jaunsen et al. (2008), Krühler et al. (2008), Perley et al. (2011b), Greiner et al. (2011), and Zafar et al. (2011), respectively. GROND observations of the afterglows of GRB 081109 and GRB 100621A are not presented elsewhere and are briefly described in the following.

\subsubsection{GRB 081109}

Swift triggered on GRB 081109 (Immler et al. 2008), and X-ray and NIR detections of the afterglow were reported by Beardmore et al. (2008) and D'Avanzo et al. (2008) soon afterwards. GROND observations were performed in seven optical/NIR filters $\left(g^{\prime} r^{\prime} i^{\prime} z^{\prime} J H K_{\mathrm{s}}\right)$ simultaneously and started 17.1 $\mathrm{hr}$ after the GRB trigger (Clemens et al. 2008b). A preliminary analysis of the spectral energy distribution (SED) revealed significant reddening of the optical/NIR afterglow (Clemens et al. 2008a). Observations of the transient with GROND continued at 2, 3, 6, and 378 days after the trigger, where the host brightness was derived from the last epoch. The GROND afterglow and host measurements are given in Table 1, and Tables 3, and 4 , respectively. A possible host galaxy was also reported in the UVOT white filter (Kuin \& Immler 2008). High-energy prompt and afterglow data, early NIR imaging, and a light curve and an $\mathrm{X}$-ray spectral analysis of this burst are presented by Jin et al. (2009).

\subsubsection{GRB 100621A}

GROND reacted immediately (Updike et al. 2010) to the Swift trigger of GRB 100621A (Ukwatta et al. 2010b) taking the first images $230 \mathrm{~s}$ after the burst. Simultaneous imaging in $g^{\prime} r^{\prime} i^{\prime} z^{\prime} J H K_{\mathrm{s}}$ continued for $3.05 \mathrm{~h}$, and was resumed on nights 2, 4 , and 10 after the burst. Analysis of the Swift/X-ray data and additional detections of the NIR afterglow were reported by Stratta et al. (2010) and Naito et al. (2010), respectively. Early GROND data found evidence of substantial reddening and host emission dominating the flux in the bluest filters, which was also seen by Swift/UVOT (Oates \& Ukwatta 2010). Afterglow and host measurements are again shown in Tables 1, 3, and 4. The temporal evolution of the optical/NIR afterglow is complex with a very steep increase in brightness of around $1.5 \mathrm{mag}$ in the $J$ band from 3.5 to $4.5 \mathrm{ks}$ after the trigger. The light curve is hence very similar to the one of GRB 081029 (Nardini et al. 2011), where an analogous behavior could be associated with the intrinsic properties of the GRB and not to changes in the intervening dust content. For completeness, the $J$-band light curve of GRB 100621A 


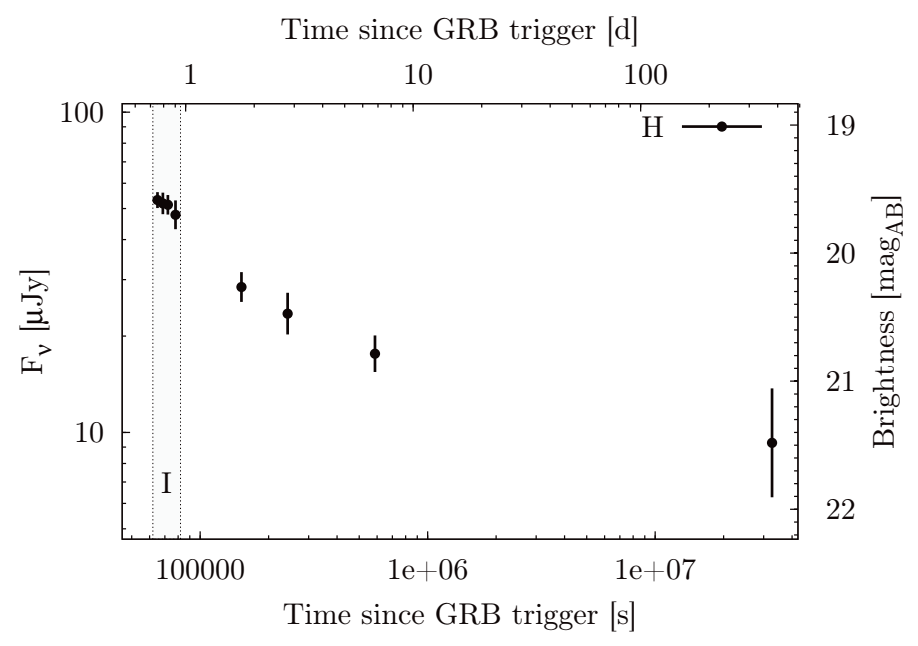

Fig. 2. GROND $H$-band light curve of the afterglow of GRB 081109 The time interval used to extract a simultaneous, host-subtracted afterglow SED from the GROND and Swift/XRT data is indicated with a grey-shaded area and labeled with I.

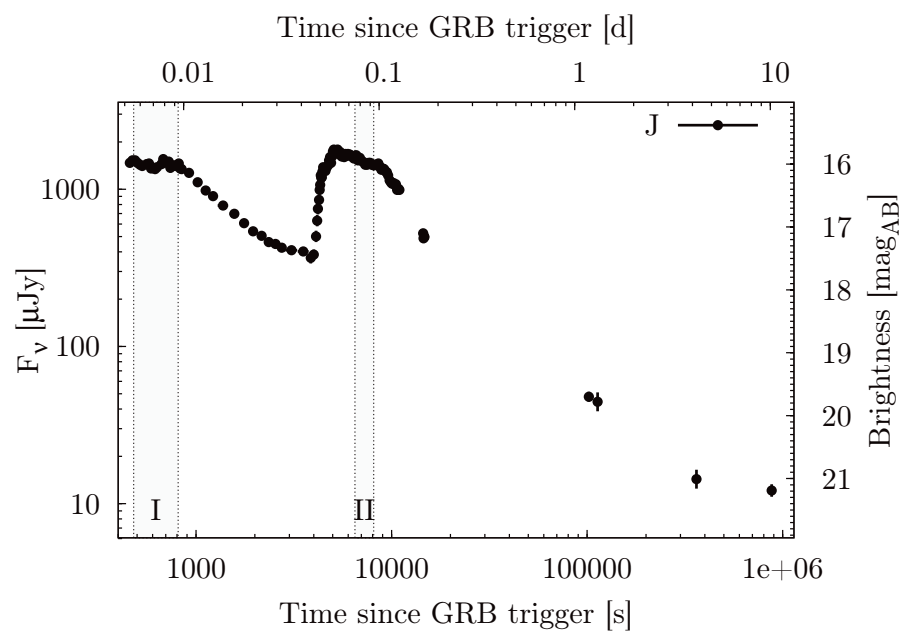

Fig. 3. GROND $J$-band light curve of the afterglow of GRB 100621A. The time intervals used to extract simultaneous, host-subtracted afterglow SEDs from the GROND and Swift/XRT data are indicated with grey-shaded areas and labeled with I and II.

is shown in Fig. 3, but its detailed modeling and interpretation is beyond the scope of this paper.

\subsection{Hosts}

Once the sample based on afterglow host extinction was defined, late follow-up observations were initiated first with GROND, and in the case of non-detections in individual filters, were continued with telescopes of successively increasing aperture size, specifically with EFOSC/SOFI at the NTT (4 m class) and FORS2/HAWKI at the VLT (8 $\mathrm{m}$ class). In one case without published redshift (GRB 081109), the photometric imaging was complemented by low-resolution spectroscopy with FORS2. Public VLT data for known hosts (GRBs 070306 and 070802) were obtained from the ESO archive. Ground-based data were complemented by Swift/UVOT imaging for GRBs 081109 and 100621A. In the case of GRB 080607, all host measurements were taken from Chen et al. (2010, 2011).

\section{Data reduction and SED fitting}

\subsection{Swift/XRT \& UVOT data}

$\mathrm{X}$-ray data were retrieved from the HEASARC archive $^{3}$, and reduced with the xrtpipeline task. Spectra were grouped to yield a minimum of 20 counts per bin, while the light curve was taken from the Swift/XRT light curve repository (Evans et al. 2007, 2009). Swift/UVOT photometry was derived following Poole et al. (2008) and is presented in Table 2.

\subsection{Ground-based optical/NIR photometry}

All optical (GROND, EFOSC, FORS1/2) and near-infrared (GROND, SOFI, HAWK-I, ISAAC) imaging was reduced in a standard manner using pyraf/IRAF (Tody 1993) similar to the procedure outlined in Krühler et al. (2008). For afterglow and host photometry, point-spread function (PSF) fitting and aperture photometry was used, respectively. The aperture diameter for individual hosts ranges typically between $1.0^{\prime \prime}$ and $2.5^{\prime \prime}$, corresponding to values between 1.5 to 4 times the PSF FWHM. It was chosen to be sufficiently large to include the largest fraction of host flux, and given the typical extent of these galaxies $\left(\lesssim 1^{\prime \prime}\right.$, i.e., $\lesssim 8.5 \mathrm{kpc}$ at $z \sim 1.5)$, the fraction of missed low-surface brightness emission is very likely not to be a major contribution to the presented measurements.

The PSF and aperture photometry was then flux calibrated using GROND observations of SDSS fields (Abazajian et al. 2009) taken immediately before or after the GRB field for the optical $g^{\prime} r^{\prime} i^{\prime} z^{\prime}$ filters. $B V R I$ photometry was obtained by creating a set of 20-30 secondary standards from the GROND photometry of field stars and the color terms from Lupton ${ }^{4}$. The $U$ band photometry was tied to observations of Landolt standard stars (Landolt 1992) taken during the same night at different airmasses, which allowed a reliable correction of the atmospheric extinction to be applied.

NIR photometry of our afterglows and hosts was calibrated with respect to 20-60 point sources in the 2MASS catalog (Skrutskie et al. 2006) in the $10^{\prime} \times 10^{\prime}$ field of view of GROND. The zeropoint for the NIR imagers with smaller field of views (in particular SOFI and ISAAC) was then derived using 3-10 secondary standards common to the GROND and SOFI/ISAAC/HAWK-I frames. The HAWK-I $Y$-band imaging was measured using the $z^{\prime}$ and $J$-band photometry from field stars using the color term

$Y=0.05+0.463 \times(z-J)+J$,

where all magnitudes are in the AB system. This color term was derived from synthetic photometry of stars with the templates of Pickles (1998) and Chabrier et al. (2000), and yields an rms residual scatter for individual stars of $0.07 \mathrm{mag}$.

This procedure resulted in typical absolute accuracies of $2-$ $5 \%$ for the optical $\left(U\right.$ to $\left.z^{\prime}\right)$ filters and $4-8 \%$ in the NIR $\left(Y J H K_{\mathrm{s}}\right)$, which were added in quadrature to the error introduced by photon noise. All data used in the analysis were corrected for the expected Galactic foreground extinction according to Schlegel et al. (1998) with $R_{\mathrm{V}}=3.08$. For the selected fields, this correction is small, i.e., $E_{B-V} \lesssim 0.15 \mathrm{mag}$ in all cases. The uncertainty of about $10 \%$ in the Galactic foreground correction

\footnotetext{
3 http://heasarc.gsfc.nasa.gov/cgi-bin/W3Browse/ swift.pl

4 http://www.sdss.org/dr7/algorithms/ sdssUBVRITransform.html
} 
Table 1. Afterglow photometric measurements.

\begin{tabular}{lcccccccc}
\hline \hline GRB & $\Delta t[\mathrm{ks}]$ & $g^{\prime}$ & $r^{\prime}$ & $i^{\prime}$ & $z^{\prime}$ & $J$ & $H$ & $K_{\mathrm{s}}$ \\
\hline 081109 & 65 & $>24.53$ & $>24.75$ & $23.6(4)$ & $22.8(3)$ & $21.24(17)$ & $19.78(11)$ & $18.83(08)$ \\
$100621 \mathrm{~A}$ & 7.6 & $21.56(17)$ & $19.92(08)$ & $18.67(06)$ & $17.71(04)$ & $16.02(04)$ & $15.02(05)$ & $14.32(06)$ \\
\hline
\end{tabular}

Notes. $\Delta t$ is the mean time of the observation after the GRB trigger. All magnitudes are in the AB system and uncorrected for Galactic foreground reddening. Values in brackets correspond to photometric errors in units of valid digits. Upper limits are given at $3 \sigma$ confidence.

Table 2. Swift/UVOT UV (uvw2 to $u$ ) photometric measurements of GRB hosts.

\begin{tabular}{lcccc}
\hline \hline Host & $u v w 2$ & $u v m 2$ & $u v w 1$ & $u$ \\
\hline GRB 081109 & $>24.1$ & $23.6(3)$ & $23.4(3)$ & $>22.9$ \\
GRB 100621A & $22.31(04)$ & $22.23(06)$ & $22.20(07)$ & $21.95(06)$ \\
\hline
\end{tabular}

Notes. All measurements in the AB system and uncorrected for the Galactic foreground reddening. Values in brackets correspond to photometric errors in units of valid digits. Upper limits are given at $3 \sigma$ confidence.

Table 3. Optical ( $U$ to $\left.z^{\prime}\right)$ photometric measurements of GRB hosts.

\begin{tabular}{lcccccccc}
\hline \hline Host & $U$ & $g^{\prime}$ & $V$ & $r^{\prime}$ & $R$ & $i^{\prime}$ & $I$ & $z^{\prime}$ \\
\hline GRB 070306 & - & $22.90(09)$ & - & $23.08(09)$ & $23.00(09)$ & $22.81(13)$ & - & $22.86(17)$ \\
GRB 070802 & - & - & - & - & $25.20(09)$ & $25.5(3)$ & - & - \\
GRB 080605 & - & $23.15(07)$ & - & $22.82(07)$ & - & $22.81(08)$ & - & $22.76(11)$ \\
GRB 080805 & - & - & $25.7(2)$ & - & $25.5(2)$ & $25.7(4)$ & - & - \\
GRB 081109 & $23.23(14)$ & $23.07(07)$ & $22.85(06)$ & $22.74(07)$ & - & $22.01(08)$ & $21.96(09)$ & $21.99(09)$ \\
GRB 090926B & $23.71(13)$ & $23.31(07)$ & - & $22.96(06)$ & - & $22.92(12)$ & - & $22.44(10)$ \\
GRB 100621A & $21.95(10)$ & $21.86(06)$ & - & $21.48(06)$ & - & $21.15(06)$ & - & $21.46(06)$ \\
\hline
\end{tabular}

Notes. All magnitudes in the AB system and uncorrected for the Galactic foreground reddening. Values in brackets correspond to photometric errors in units of valid digits. Upper limits are given at $3 \sigma$ confidence. Data for the host of GRB 080607 were taken from Chen et al. (2010, 2011), and are not shown in the table.

Table 4. NIR ( $Y$ to $K$ ) photometric measurements of GRB hosts.

\begin{tabular}{lccccccc}
\hline \hline Host & $Y$ & $J_{\text {GROND }}$ & $J$ & $H_{\text {GROND }}$ & $H$ & $K_{\text {GROND }}$ & $K$ \\
\hline GRB 070306 & - & $21.9(4)$ & $21.62(08)$ & $21.5(4)$ & $21.20(12)$ & $>21.1$ & $21.38(10)$ \\
GRB 070802 & - & - & $24.5(3)$ & - & - & - & $23.4(3)$ \\
GRB 080605 & - & $21.9(2)$ & - & $22.3(3)$ & - & $>21.1$ & - \\
GRB 080805 & - & - & $23.6(2)$ & - & - & - & $23.1(2)$ \\
GRB 081109 & $21.63(08)$ & $21.40(17)$ & $21.37(06)$ & $21.5(4)$ & - & $>20.6$ & $21.05(08)$ \\
GRB 090926B & - & $22.3(4)$ & $21.88(13)$ & $>21.6$ & $21.9(3)$ & $>20.9$ & $21.44(19)$ \\
GRB 100621A & $21.10(06)$ & $21.22(10)$ & $21.43(06)$ & $21.18(14)$ & - & $>21.1$ & $21.23(11)$ \\
\hline
\end{tabular}

Notes. All measurements are given in the AB system and are uncorrected for the Galactic foreground reddening. Values in brackets correspond to photometric errors in units of valid digits. Upper limits are given at $3 \sigma$ confidence.

does therefore not affect the overall results of this work. Groundbased photometric measurements of the afterglows and hosts are shown in Tables 1,3 , and 4, respectively.

\subsection{Long-slit spectroscopy}

In addition to the photometric observations, the host of GRB 081109 was also observed spectroscopically with FORS2 (Appenzeller et al. 1998) at the VLT. In total, $2 \times 1200 \mathrm{~s}$ spectra were obtained with the grisms $300 \mathrm{~V}$ and $300 \mathrm{I}$ and a long-slit width of $1.6^{\prime \prime}$. Acquisition images were taken in the $V$ and $I$ filters. The spectroscopic data were obtained at airmasses of $\sim 1.1$ and seeing of $0.9^{\prime \prime}$, which results in a line-spread function of approximately $1.9 \mathrm{~nm}$ at $570.0 \mathrm{~nm}$.

The data were reduced using standard procedures in pyraf/IRAF, with the wavelength solution obtained against an $\mathrm{HeHgCd}$ arclamp exposures with 25 lines leaving residuals of around $0.07 \mathrm{~nm}$ rms. Flux-calibration was performed against the spectrophotometric standard BPM162745. The wavelengthand flux-calibrated spectrum was corrected for Galactic foreground extinction and renormalized to the available braod-band photometry.

\subsection{SED fitting}

\subsubsection{Afterglows}

For the afterglow SED analysis, X-ray (0.3-10 keV) and opti$\mathrm{cal} /$ NIR data were fit together under the assumption that the underlying continuum emission is well-represented by synchrotron emission (e.g., Sari et al. 1998; Galama \& Wijers 2001; Schady et al. 2007; Rossi et al. 2011a).

\footnotetext{
5 http://www.eso.org/sci/observing/tools/standards/ spectra/bpm16274.html
} 
Rest-frame soft X-ray photons are absorbed by metals, predominantly $\alpha$-chain elements, while UV over optical to NIR wavelengths are decreasingly affected by dust absorption. Good coverage above $1 \mathrm{keV}$ combined with NIR observations allows for an accurate determination of the continuum, and hence good constraints on the dust abundance (represented by the absorption in the rest-frame, optical $V$-band, $A_{\mathrm{V}}^{\mathrm{GRB}}$ ), the extinction law and total metal content (converted into a hydrogen-equivalent column density $N_{\mathrm{H}, \mathrm{X}}$ assuming solar abundances from Anders \& Grevesse 1989) along the line of sight. In particular, single and broken power-law continua were used, where in the latter case the two power-law slopes $\beta_{1}$ and $\beta_{2}$ were fixed to yield $\beta_{1}+0.5=\beta_{2}$, as expected for the cooling break of synchrotron emission in the slow cooling regime (e.g., Sari et al. 1998; Granot \& Sari 2002).

The NIR to X-ray SEDs were fitted in X-spec (Arnaud 1996) using extinction laws for the Milky Way (MW), and both Small (SMC) and Large (LMC) Magellanic Clouds from Pei (1992). For the GROND photometry, measurements from the time frame indicated in Figs. 2 and 3 were used, where we chose interval II for GRB 100621A owing to the higher signal-to-noise ratio of these data. Data taken at interval I yield consistent results for $A_{\mathrm{V}}^{\mathrm{GRB}}$ and $N_{\mathrm{H}, \mathrm{X}}$. In the X-ray regime, where spectral changes in the late evolution of an afterglow are typically very moderate or in most cases even completely absent, the full XRT data set with a constant hardness ratio was used to create a time-averaged spectrum. The latter was then rescaled to the flux value at the time of the GROND observations derived from fitting the XRT light-curve with simple afterglow models. The early steep decay of the X-ray light curve and epochs of flaring activity were excluded from the combined spectrum as well as the light-curve fitting.

\subsubsection{Hosts}

The UV/optical/NIR photometry of the hosts of the selected GRBs were analyzed in a standard way using stellar population synthesis (SPS) techniques to convert luminosities into stellar masses $M_{*}$ (e.g., Bell et al. 2003; Ilbert et al. 2010) within LePhare $^{6}$. In detail, $3 \times 10^{6}$ galaxy templates based on models from Bruzual \& Charlot (2003) with a universal IMF (Chabrier 2003) and different ages, star formation histories, extinction laws, reddening values and metallicities were fit to the data. In addition, emission lines were taken into account by converting the de-reddened UV luminosity into a star formation rate, and line strengths of $\mathrm{Ly}-\alpha, \mathrm{H}_{\alpha}, \mathrm{H}_{\beta}$, [OII], and [OIII], which were determined following Kennicutt (1998) and Ilbert et al. (2009). In particular, for vigorously star-forming galaxies such as GRB hosts, this effect is a significant contribution to even broad-band photometry (e.g., Watson et al. 2011) and reaches values of up to $\sim 0.2-0.3 \mathrm{mag}$ (Ilbert et al. 2009). To permit a direct comparison with results published in the literature (e.g., Fontana et al. 2006; Marchesini et al. 2009; Ilbert et al. 2010, SGL09), we used the attenuation law of Calzetti et al. (2000) derived for starburst galaxies in the fitting, unless different reddening laws provided a closer fit to the host data at $90 \%$ confidence. We caution that access to the rest-frame NIR, which enables the most reliable measurement of the stellar mass $M_{*}$ of a galaxy (see e.g., Castro Cerón et al. 2010, and references therein), is somewhat limited for part of the sample. However, all hosts are detected in at least one filter redwards of the $4000 \AA$ break, which allows a

\footnotetext{
${ }^{6}$ http://www . cfht . hawaii. edu/ arnouts/LEPHARE
}

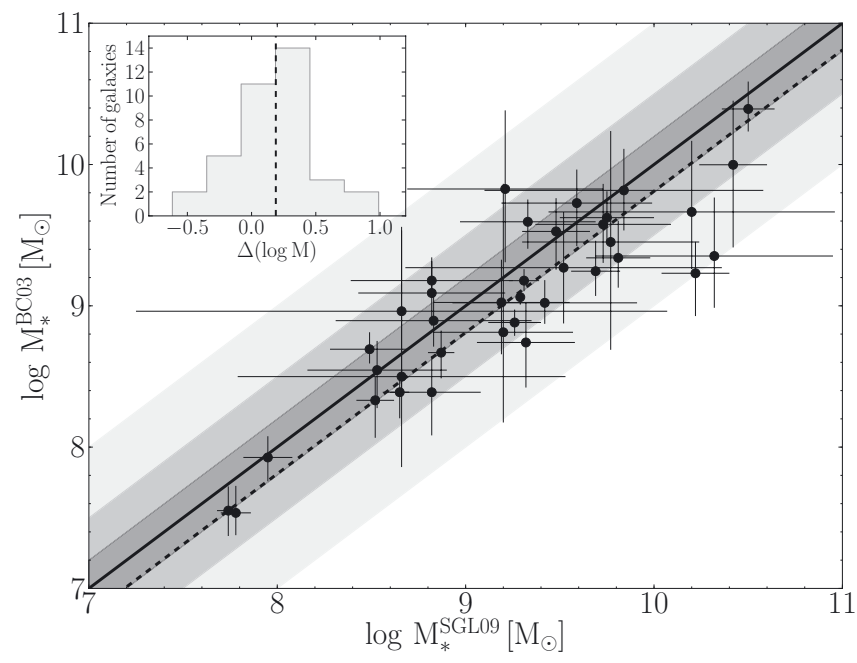

Fig. 4. Comparison of stellar masses from the host sample of SGL09. The plot shows host masses derived from the photometry compiled in SGL09 and following Sect. 4.4.2 with models from Bruzual \& Charlot (2003) against values directly taken from SGL09. Error bars are the maximum and minimum values of the stellar mass in the global $\chi^{2}$ distribution of $3 \times 10^{6}$ galaxy templates (see Sect. 4.4.2). The solid lines represents equality, and the dashed line the median offset. Increasingly gray shaded areas indicate dispersions of $0.2,0.5$, and 1.0 dex, respectively. The inset shows the distribution of mass differences, which has a median of around 0.2 dex.

reasonable estimate of $M_{*}$ for a galaxy (e.g., Glazebrook et al. 2004; Ilbert et al. 2009, SGL09).

Systematic uncertainties of up to an average of $0.2-0.3 \mathrm{dex}$ in $M_{*}$ are caused by the specific details of the stellar population models and the assumed attenuation/extinction law (Pozzetti et al. 2007; Küpcü Yoldaş et al. 2007; Kajisawa et al. 2009; Ilbert et al. 2010). Despite the small sample size of GRB hosts, there is evidence for an offset of around 0.2 dex between the presented method and the one of SGL09 as shown in Fig. 4. In the following, the recalculated stellar masses of the long GRB hosts with the photometric data compiled in SGL09 are used as a comparison sample.

The SPS fit returns not only the luminosity and mass of the galaxy, but also other physical properties of the host, such as the age of the dominant stellar population $\tau$, its color-excess $E_{(B-V)}$, and the star-formation rate (SFR) derived from the rest-frame UV flux. Reported physical host properties are the median of the probability distribution of the total grid over all galaxy templates at the fixed spectroscopic redshift. Errorbars represent the maximum and minimum values of the respective parameter in the global $\chi^{2}$-distribution of the multi-dimensional parameter grid. Typically, errors are asymmetric and dominated by the uncertainty in the color excess in the host galaxy, which results in logarithmic errors in all galaxy parameters. Absolute magnitudes and masses are compared with the redshift-dependent galaxy luminosity (Willmer et al. 2006; Marchesini et al. 2007) and stellar mass functions (Marchesini et al. 2009; Ilbert et al. 2010)

\section{Results}

\subsection{Afterglow extinction and metal-to-dust ratios}

Of the total of eight afterglows in the sample, the extinction properties of five of them (GRBs 070802, 080605, 080607, 080805, 090926B) were extensively discussed in previous studies (Krühler et al. 2008; Elíasdóttir et al. 2009; Perley et al. 2011b; Greiner et al. 2011; Zafar et al. 2011). In these cases, 
Table 5. Afterglow properties.

\begin{tabular}{|c|c|c|c|c|c|c|c|}
\hline Afterglow & Redshift* & $\begin{array}{c}T_{90}{ }^{* *} \\
{[\mathrm{~s}]} \\
\end{array}$ & $\beta_{\mathrm{opt}}{ }^{* * *}$ & $\beta_{\mathrm{X}}$ & $\begin{array}{c}A_{V}^{\mathrm{GRB}} \\
{[\mathrm{mag}]} \\
\end{array}$ & $\begin{array}{c}N_{\mathrm{H}, \mathrm{X}} \\
10^{22} \mathrm{~cm}^{-2} \\
\end{array}$ & $\chi^{2} /$ d.o.f. \\
\hline GRB 070306 & 1.496 & $210^{(\mathrm{a})}$ & 1.00 & $1.00 \pm 0.07$ & $5.5_{-1.0}^{+1.2}$ & $2.5_{-0.2}^{+0.3}$ & $123 / 108$ \\
\hline GRB $070802^{(\mathrm{b}, \mathrm{c})}$ & 2.452 & $16.4^{(\mathrm{d})}$ & 0.60 & $1.10_{-0.12}^{+0.14}$ & $1.23_{-0.16}^{+0.18} / 1.19 \pm 0.15$ & $2.0_{-0.8}^{+0.7} /<2.9$ & $15 / 14$ \\
\hline GRB $080605^{(\mathrm{b}, \mathrm{c})}$ & 1.640 & $20^{(\mathrm{e})}$ & 0.67 & $0.67 \pm 0.01$ & $0.47 \pm 0.03 / 1.20_{-0.10}^{+0.09}$ & $1.01_{-0.08}^{+0.09} / 0.71 \pm 0.08$ & $428 / 327$ \\
\hline GRB $080607^{(\mathrm{c}, \mathrm{f})}$ & 3.036 & $79^{(\mathrm{g})}$ & 0.96 & $0.96_{-0.06}^{+0.05}$ & $3.3 \pm 0.3 / 2.33_{-0.43}^{+0.46}$ & $2.7_{-0.7}^{+0.8} / 3.8_{-0.2}^{+0.2}$ & $70 / 39$ \\
\hline GRB $080805^{(\mathrm{b}, \mathrm{c})}$ & 1.505 & $78^{(\mathrm{h})}$ & 0.47 & $0.97 \pm 0.05$ & $1.01_{-0.14}^{+0.19} / 1.53 \pm 0.13$ & $1.0_{-0.4}^{+0.6} / 1.2_{-0.5}^{+0.4}$ & $23 / 18$ \\
\hline 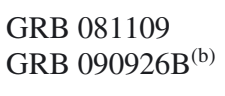 & $\begin{array}{c}0.979 \\
1.24\end{array}$ & $\begin{array}{l}190^{(\mathrm{i})} \\
110^{(\mathrm{j})}\end{array}$ & $\begin{array}{l}1.10 \\
0.73\end{array}$ & $\begin{array}{l}1.12_{-0.02}^{+0.02} \\
0.73_{-0.07}^{+0.09}\end{array}$ & $\begin{array}{l}3.4_{-0.3}^{+0.4} \\
1.4_{-0.6}^{+1.1}\end{array}$ & $\begin{array}{c}1.10_{-0.12}^{+0.13} \\
2.2_{-0.4}^{+0.5}\end{array}$ & $\begin{array}{l}48 / 63 \\
33 / 31\end{array}$ \\
\hline GRB $100621 \mathrm{~A}$ & 0.542 & $63.6^{(\mathrm{k})}$ & 0.79 & $1.29_{-0.10}^{+0.11}$ & $3.8_{-0.2}^{+0.2}$ & $1.62_{-0.15}^{+0.15}$ & $138 / 124$ \\
\hline
\end{tabular}

Notes. Afterglow measurements were taken from the reference denoted with the superscript in the first line. When two references are given, we quote both values for $A_{V}^{\mathrm{GRB}}$ and $N_{\mathrm{H}, \mathrm{X}}$, but values for $\beta$ and $\chi^{2}$ only from the first one for the sake of clarity. ${ }^{(*)}$ Redshifts from Jaunsen et al. (2008), Elíasdóttir et al. (2009), Fynbo et al. (2009a), Fynbo et al. (2009b) and Milvang-Jensen et al. (2010). ${ }^{(* *)} T_{90}$ is the duration in which the GRB emits from 5\% to $95 \%$ of its $\gamma$-rays, and is used to discriminate between short and long bursts. Typically, long GRBs have $T_{90}>2 \mathrm{~s}$ (Kouveliotou et al. 1993). All GRBs in this work are hence unambigously long events. ${ }^{(* *)} \beta_{\text {opt }}$ is tied to $\beta_{X}$ in the fitting, and hence has the same error.

References. (a) Barthelmy et al. (2007), (b) Greiner et al. (2011), (c) Zafar et al. (2011), (d) Cummings et al. (2007), (e) Cummings et al. (2008), (f) Perley et al. (2011b), (g) Stamatikos et al. (2008), (h) Palmer et al. (2008), (i) Markwardt et al. (2008), (j) Baumgartner et al. (2009), (k) Ukwatta et al. (2010a).

the published afterglow analysis is comparable to the approach adopted in this work, and its description is therefore not repeated here, with results from the literature being summarized in Table 5. For the remaining three events (GRBs 070306, 081109, $100621 \mathrm{~A})$, we present either new data and their modeling (GRBs $081109,100621 \mathrm{~A})$, or a new analysis (GRB 070306) in the following.

The afterglow of GRB 070306 was discussed in Jaunsen et al. (2008). As their analysis differs significantly from our approach, we refit the available afterglow data following Sect. 4.4.1. The broad-band SED in Fig. 5 is reasonably well fit $\left(\chi^{2}=123 / 108\right.$ d.o.f.) with a single power-law continuum of spectral index $\beta=1.00 \pm 0.07$, an $A_{\mathrm{V}}^{\mathrm{GRB}}=5.5_{-1.0}^{+1.2} \mathrm{mag}$, and an $N_{\mathrm{H}, \mathrm{X}}=$ $2.5_{-0.2}^{+0.3} \times 10^{22} \mathrm{~cm}^{-2}$ at $90 \%$ confidence, which implies a metalto-dust ratio of $N_{\mathrm{H}, \mathrm{X}} / A_{\mathrm{V}}^{\mathrm{GRB}}=4.4 \times 10^{21} \mathrm{~cm}^{-2} / \mathrm{mag}$. Given the redshift of $z=1.496$, and the sparse wavelength coverage in the NIR (probing the rest-frame optical redward of $400 \mathrm{~nm}$, where there is little distinction between local extinction laws), all local dust models of course provide equally good fits to the data, and within errors compatible values of $\beta, A_{\mathrm{V}}^{\mathrm{GRB}}$, and $N_{\mathrm{H}, \mathrm{X}}$. No strong statements can be made about the presence of a break between the NIR and X-ray data. We adopt the model with the least number of free parameters (single power-law continuum, which also yields the lowest $\chi^{2}$ ), but note that in the case of a break between the two wavelength regimes (as seen in most early GRB afterglows, Greiner et al. 2011) the fit is of comparable quality $\left(\Delta \chi^{2}=3\right)$, and yields a best-fit $A_{\mathrm{V}}^{\mathrm{GRB}}$ that is significantly lower $\left(A_{\mathrm{V}}^{\mathrm{GRB}}=4.3_{-1.0}^{+1.1} \mathrm{mag}\right)$, but within the errors consistent with the single power-law values.

The afterglow SED for GRB 081109 at $z=0.979$ was constructed from GROND and Swift data and is shown in Fig. 6. After subtraction of the late host epoch, no residual flux was detected in the two bluest GROND filters $g^{\prime}$ and $r^{\prime}$. The afterglow was detected in all five redder bands, which implies an extremely red color of $(R-K)_{\mathrm{AB}} \gtrsim 6$ mag and $\beta_{\mathrm{oX}}<0.44$. The combined GROND and XRT data are closely fitted with a single power-law continuum, indicating that both the optical/NIR

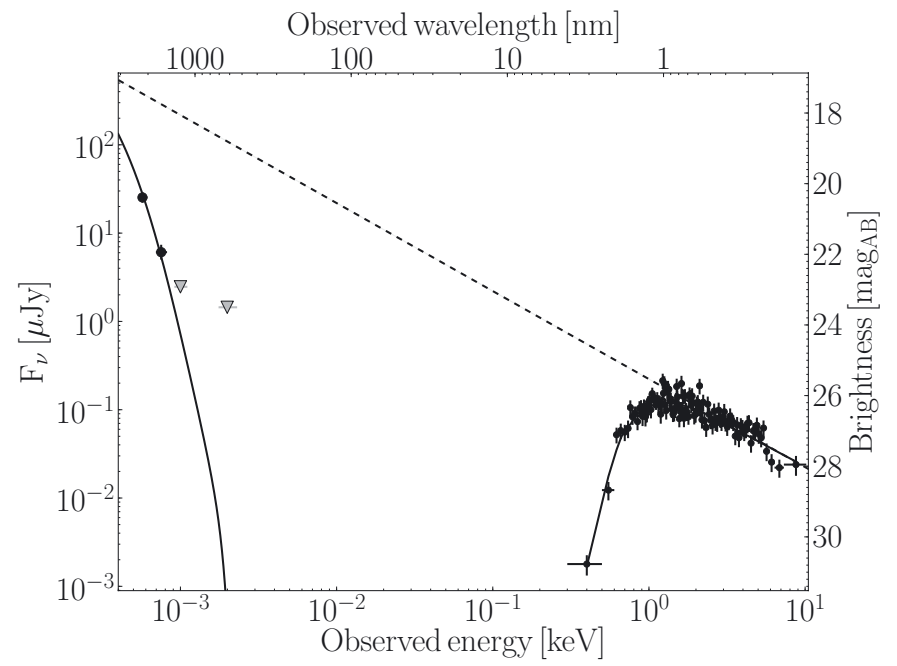

Fig. 5. NIR to X-ray SED of the afterglow of GRB 070306 obtained approximately $120 \mathrm{ks}$ after the trigger in the observer's frame. The dashed line shows the unabsorbed synchrotron continuum emission while the best-fit model (including dust and metal absorption) is shown by solid lines. Upper limits are shown by downward triangles.

and the X-ray regimes probe the same part of the synchrotron spectrum. The obvious curvature in the GROND data is accurately described with either of the local dust models, with bestfit parameters of $A_{\mathrm{V}}^{\mathrm{GRB}}=3.4_{-0.3}^{+0.4} \mathrm{mag}, \beta=1.12 \pm 0.02$, and $N_{\text {H.X }}=(1.1 \pm 0.1) \times 10^{22} \mathrm{~cm}^{-2}$ at $90 \%$ confidence and a $\chi^{2}$ of 48.1 for 63 d.o.f. in a MW-like extinction law. The SMC and LMC models yield within the errors comparable parameters and provide equally good fits to the data $\left(\chi^{2} \mathrm{~s}\right.$ of 48.2 and 48.8 , respectively).

The SED of the afterglow of GRB 100621A at $z=0.542$ (Milvang-Jensen et al. 2010) is shown in Fig. 7. As for the SED of GRB 081109, there is strong curvature and obvious reddening in the optical/NIR part of the SED. The inferred ultra-red 


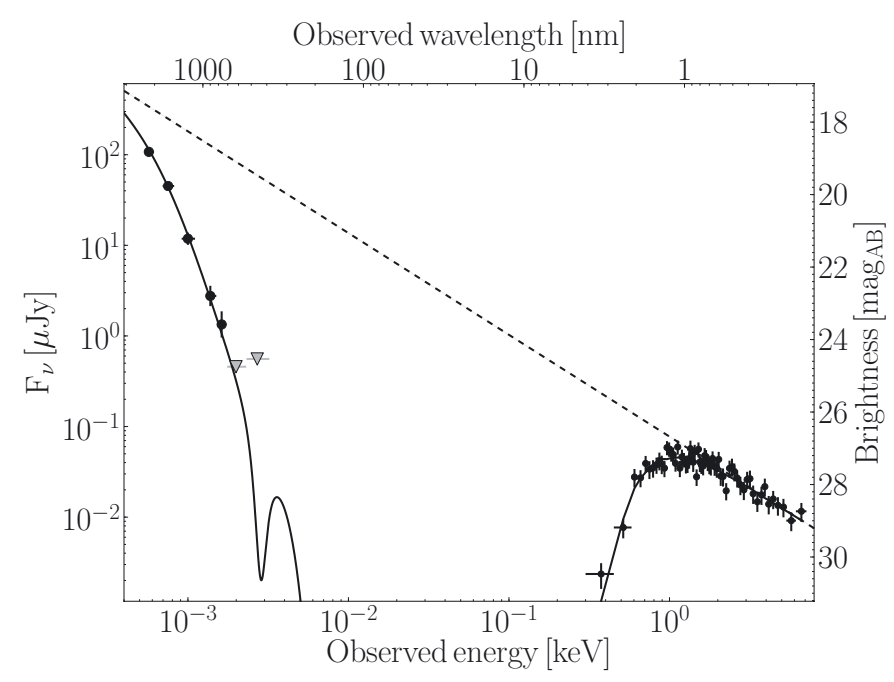

Fig. 6. Same as Fig. 5 for the afterglow of GRB 081109 obtained approximately $60 \mathrm{ks}$ after the trigger.

color of $(R-K)_{\mathrm{AB}} \sim 5.8 \mathrm{mag}$, and the $\beta_{\mathrm{OX}}$ value of 0.39 provide evidence of strong dust extinction. The best-fit solution is obtained with a broken power-law with spectral indices $\beta_{1}=\beta_{2}-0.5=0.79_{-0.10}^{+0.11}$, as well as $A_{\mathrm{V}}^{\mathrm{GRB}}=3.8 \pm 0.2 \mathrm{mag}$ for an LMC-like extinction law, and $N_{\text {H.X }}=(1.62 \pm 0.15) \times 10^{22} \mathrm{~cm}^{-2}$ at $90 \%$ confidence ( $\chi^{2}$ of 138 for 124 d.o.f.). Given the rest-frame wavelength coverage of $\sim 300-1500 \mathrm{~nm}$, all local dust models return comparable values with $A_{\mathrm{V}}^{\mathrm{GRB}}$ values of $3.8 \pm 0.2$ mag for an SMC- and 4.0 \pm 0.2 mag for a MW-type extinction law. All data bluewards of and including the $r^{\prime}$ filter are consistent with these extinction laws, while the $g^{\prime}$-band photometry is somewhat $(2-3 \sigma)$ brighter than the best-fit model predicts. This could indicate a discrepancy between the details of the specific dust extinction law and local models similar to that observed e.g., in GRBs 070802 or 080607 (Elíasdóttir et al. 2009; Perley et al. 2011b).

The visual extinctions of GRBs 070306, 081109, and $100621 \mathrm{~A}$ are among the largest ever measured directly along GRB sight-lines, and imply metal-to-dust ratio of $N_{\mathrm{H}, \mathrm{X}} / A_{\mathrm{V}}^{\mathrm{GRB}} \sim$ $(3-5) \times 10^{21} \mathrm{~cm}^{-2} / \mathrm{mag}$. Compared to previous afterglow measurements, these $N_{\mathrm{H}, \mathrm{X}} / A_{\mathrm{V}}^{\mathrm{GRB}}$ values are relatively low, and within a factor of 2-3 similar to $N_{\mathrm{H}} / A_{\mathrm{V}}$ as observed in the SMC, LMC, and MW (see also Sect. 6.4). The results of afterglow SED fitting, as well as values taken from the literature, are summarized in Table 5.

\subsection{Host properties}

\subsubsection{The host of GRB 070306}

The galaxy hosting the strongly extinguished GRB 070306 at $z=1.496$ was previously discussed in Jaunsen et al. (2008). In addition to the public VLT imaging data (FORS $R$, ISAAC $J_{\mathrm{s}} H K_{\mathrm{s}}$ ), the host of GRB 070306 was observed with GROND ( $g^{\prime} r^{\prime} i^{\prime} z^{\prime} J H K_{\mathrm{S}}$ simultaneously), and its SED (Fig. 8) was complemented with published $u$ and $I$-band data (Jaunsen et al. 2008). The host is bright $\left(r^{\prime}=23.1 \mathrm{mag}\right)$, mildly $\operatorname{red}^{7}$ with $(R-K)_{\mathrm{AB}} \sim$ $1.5 \mathrm{mag}$, and shows evidence of a Balmer/4000 $\AA$ break. The

\footnotetext{
7 We compare GRB host colors with the median $(R-K)_{\mathrm{AB}}=0.8$ mag color from the SGL09 sample.
}

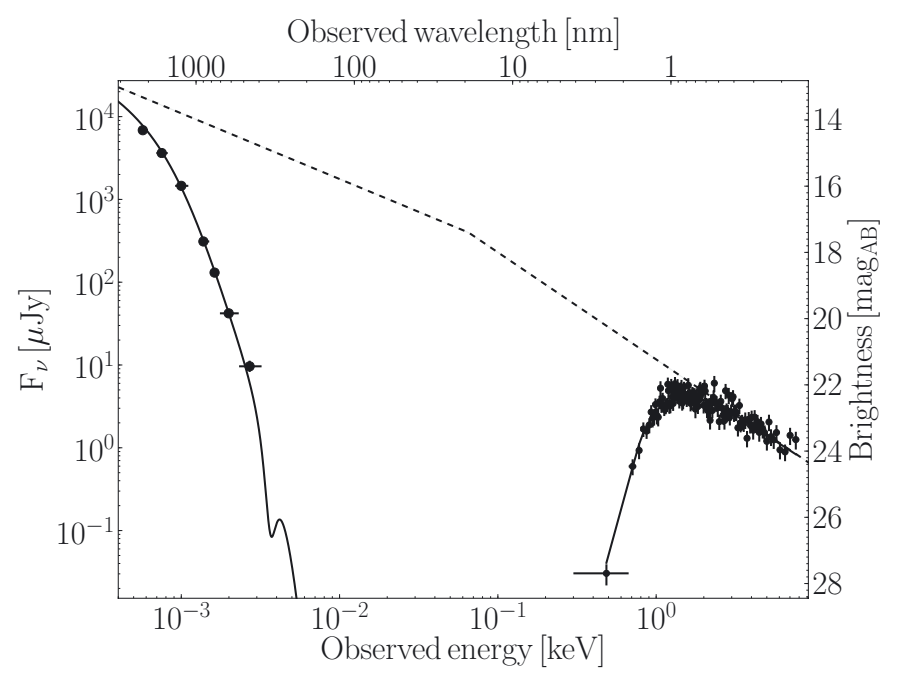

Fig. 7. Same as Fig. 5 for the afterglow of GRB 100621A obtained approximately $7.6 \mathrm{ks}$ after the trigger.

data are closely fit with a non-extinguished $\left(A_{\mathrm{V}}^{\prime}<0.6\right)^{8}$ host template, and yield an absolute $\mathrm{AB}$ magnitude of $M_{B}=-22.4 \pm$ $0.1 \mathrm{mag}$, which, at $z \sim 1.5$, corresponds to $\sim 1.7 L^{*}$. The stellar mass of $\log \left(M_{*}\left[M_{\odot}\right]\right)=10.4 \pm 0.2$ places the galaxy among the most massive hosts compared to the sample of SGL09. The star-formation rate estimate based on the rest-frame UV flux is $13_{-4}^{+11} M_{\odot} / \mathrm{yr}$, which gives a specific star formation rate $\left(S S F R=S F R / M_{*}\right)$ of $\sim 0.5 \mathrm{Gyr}^{-1}$, or growth timescale (i.e., $1 / S S F R$ ) of 2 Gyr. The SFR is in reasonable agreement with the one derived from the [OII] emission line (Jaunsen et al. 2008). We note, that the ISAAC $H$-band host image was obtained only 2.5 days after the GRB, hence very likely contains a significant fraction of afterglow light, explaining the blue $H-K$ color. The physical properties of the host, however, are comparable when determined with or without using the ISAAC $H$-band data.

\subsubsection{The host of GRB 070802}

The host of GRB 070802 at $z=2.452$ was discovered in deep FORS $R$ and ISAAC $K$ band imaging (Elíasdóttir et al. 2009). The afterglow SED is characterized by a prominent $2175 \AA$ dust feature, and significant dust in the range of $A_{\mathrm{V}} \sim 1 \mathrm{mag}$ (Krühler et al. 2008; Elíasdóttir et al. 2009). To construct the optical/NIR host SED, the $R \sim 25.2 \mathrm{mag}$ host was also observed with EFOSC/NTT in $i$ and HAWK-I/VLT in the $J$-band. The galaxy is moderately red $\left((R-K)_{\mathrm{AB}} \sim 1.8 \mathrm{mag}\right.$ ) and its SED (Fig. 8 ) shows a Balmer/4000 $\AA$ break, but the age of the dominant stellar population is not well-constrained by the available data ( $\lesssim 1 \mathrm{Gyr})$. There is no strong evidence of internal reddening, and the bestfit absolute magnitude is $M_{B}=-21.4 \pm 0.2 \mathrm{mag}$, which is $\sim 0.6 L^{*}$ at $z \sim 2.5$ with $\log \left(M_{*}\left[M_{\odot}\right]\right)=9.7_{-0.3}^{+0.2}$. Using the rest-frame UV flux derived from the galaxy model fitting, an estimate for the SFR of $10_{-7}^{+30} M_{\odot} / \mathrm{yr}$, and the SSFR of $\sim 2 \mathrm{Gyr}^{-1}$ can be derived.

${ }^{8}$ For galaxies, we use $A_{\mathrm{V}}^{\prime}$, or $E_{B-V}^{\prime}$ to indicate measured attenuation and effective reddening, since these quantities depend for example on the topology of the ISM and galaxy geometry (Pierini et al. 2004), and on galaxy scales are different from the corresponding values of a given extinction law. 
T. Krühler et al.: The SEDs and host galaxies of the dustiest GRB afterglows
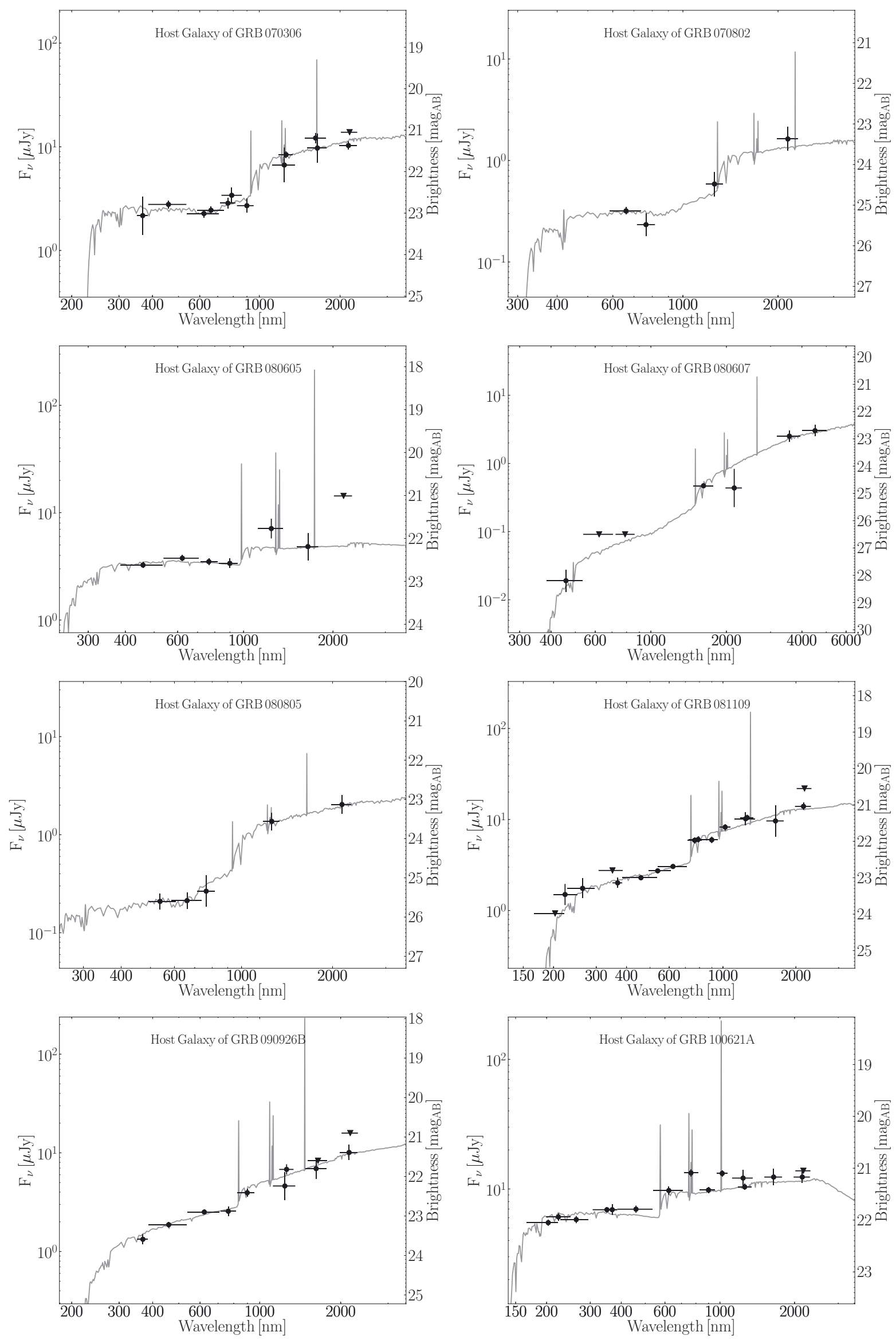

Fig. 8. SEDs of the host of in this sample and the best-fit galaxy model (solid line) in the observer frame. Filled black circles represent photometric measurements, while downward triangles denote $3 \sigma$ upper limits. 
Table 6. Host galaxy properties.

\begin{tabular}{|c|c|c|c|c|c|c|c|c|}
\hline Host & $z_{\mathrm{spec}}$ & $z_{\text {phot }}$ & $\begin{array}{l}\mathrm{RA}^{a} \\
\mathrm{~J} 2000\end{array}$ & $\begin{array}{l}\text { Dec }^{a} \\
\text { J2000 }\end{array}$ & $\begin{array}{l}\text { Offset }^{b} \\
\operatorname{arcsec}^{2}\end{array}$ & Probability $^{c}$ & Instruments $^{d}$ & Filter \\
\hline GRB 070306 & 1.496 & $1.64_{-0.13}^{+0.35}$ & 09:52:23.31 & $+10: 28: 55.4$ & $\lesssim 0^{\prime} 2$ & 0.002 & FGI & $g^{\prime} r^{\prime} R i^{\prime} z^{\prime} J_{G} J H K^{(f)}$ \\
\hline GRB 070802 & 2.452 & $2.3_{-0.6}^{+1.5}$ & $02: 27: 35.72$ & $-55: 31: 39.0$ & $0{ }^{\prime} 15 \pm 0 .{ }^{\prime} 08$ & 0.011 & EFHI & RIJK \\
\hline GRB 080605 & 1.640 & $1.7_{-0.2}^{+0.7}$ & $17: 28: 30.05$ & $+04: 00: 56.0$ & $0 ! 23 \pm 0 . ' 11$ & 0.002 & G & $g^{\prime} r^{\prime} i^{\prime} z^{\prime} J H$ \\
\hline GRB 080805 & 1.505 & $1.7_{-0.7}^{+0.6}$ & $20: 56: 53.43$ & $-62: 26: 39.3$ & $\lesssim 0^{\prime \prime} 3$ & 0.030 & $\mathrm{EH}$ & $V R I J K$ \\
\hline GRB 081109 & 0.979 & $0.84_{-0.07}^{+0.16}$ & 22:03:09.63 & $-54: 42: 39.9$ & $\lesssim 0 \prime^{\prime} 2$ & 0.002 & EFGHU & $U g^{\prime} V r^{\prime} i^{\prime} I z^{\prime} Y J_{G} J H K$ \\
\hline GRB 090926B & 1.24 & $1.43_{-0.26}^{+0.62}$ & 03:05:13.91 & $-39: 00: 22.6$ & $\lesssim 0 !^{\prime} 6$ & 0.018 & EFGS & $U g^{\prime} r^{\prime} i^{\prime} z^{\prime} J H K$ \\
\hline GRB 100621A & 0.542 & $0.50_{-0.02}^{+0.14}$ & 21:01:13.08 & $-51: 06: 22.2$ & $0{ }^{\prime} 12 \pm 0 . \prime 08$ & 0.0006 & EGHU & $w 2 m 2 w 1 u U g^{\prime} r^{\prime} i^{\prime} z^{\prime} Y J H K$ \\
\hline
\end{tabular}

Notes. Redshifts from Jaunsen et al. (2008), Elíasdóttir et al. (2009), Fynbo et al. (2009a), Fynbo et al. (2009b) and Milvang-Jensen et al. (2010). The host of GRB 080607 is not shown in this table. All measurements were directly taken from Chen et al. (2010, 2011). (a) Host position derived after tying the astrometric solution to the USNO-B1 catalog (Monet et al. 2003). Typical absolute uncertainties are $\approx 0 .{ }^{\prime} 3{ }^{(b)}$ Relative offset calculated by registering the host images against astrometric templates derived from afterglow images with a typical precision of 40 mas rms. ${ }^{(c)}$ Estimated chance coincidence probability following Bloom et al. (2002) and Perley et al. (2009). ${ }^{(d)}$ G is GROND at the $2.2 \mathrm{~m}$ MPG/ESO telescope, E/S are EFOSC/SOFI at the NTT, and F/H/I are HAWK-I, FORS1/2 and ISAAC at the VLT, and U UVOT onboard Swift respectively. ${ }^{(f)}$ Further complemented by the $u$ and $I$ band magnitudes in Cool et al. (2007) and Jaunsen et al. (2008).

Table 7. SPS host galaxy fitting results.

\begin{tabular}{lccccccccc}
\hline \hline Host & Redshift & $\begin{array}{c}M_{B} \\
\mathrm{mag}_{\mathrm{AB}}\end{array}$ & $\begin{array}{c}\mathrm{Age} \\
\mathrm{Gyr}\end{array}$ & $\begin{array}{c}E_{B-V}^{\prime} \\
\mathrm{mag}\end{array}$ & $\begin{array}{c}\log \left(M_{*}\right) \\
M_{\odot}\end{array}$ & $\begin{array}{c}\log S F R \\
M_{\odot} / \mathrm{yr}\end{array}$ & $\begin{array}{c}\log S S F R \\
\mathrm{yr}^{-1}\end{array}$ & $\begin{array}{c}L \\
L_{B}^{*}\end{array}$ & $\chi^{2} / N_{\text {filt }}$ \\
\hline GRB 070306 & 1.496 & $-22.4 \pm 0.1$ & $1.6_{-1.0}^{+1.6}$ & $<0.16(2 \sigma)$ & $10.39_{-0.15}^{+0.19}$ & $1.1_{-0.2}^{+0.3}$ & $-9.3_{-0.3}^{+0.4}$ & $1.7 \pm 0.2$ & $11.8 / 12$ \\
GRB 070802 & 2.452 & $-21.4 \pm 0.2$ & $0.38_{-0.31}^{+0.66}$ & $<0.42(2 \sigma)$ & $9.7_{-0.3}^{+0.2}$ & $1.0_{-0.5}^{+0.6}$ & $-8.7_{-0.6}^{+1.0}$ & $0.6 \pm 0.2$ & $2.3 / 4$ \\
GRB 080605 & 1.640 & $-22.6 \pm 0.2$ & $0.06_{-0.03}^{+0.18}$ & $<0.22(2 \sigma)$ & $9.6_{-0.2}^{+0.3}$ & $1.6_{-0.3}^{+0.3}$ & $-8.0_{-0.6}^{+0.5}$ & $2.1 \pm 0.4$ & $3.3 / 6$ \\
GRB 080607 & 3.036 & $-21.1 \pm 0.1$ & $0.16_{-0.12}^{+0.53}$ & $0.35_{-0.09}^{+0.25}$ & $9.9_{-0.6}^{+0.4}$ & $1.6_{-0.5}^{+0.4}$ & $-8.2_{-0.7}^{+0.6}$ & $0.3 \pm 0.1$ & $3.9 / 5$ \\
GRB 080805 & 1.505 & $-20.4 \pm 0.2$ & $0.51_{-0.40}^{+0.74}$ & $<0.65(2 \sigma)$ & $9.7_{-0.2}^{+0.2}$ & $0.8_{-0.8}^{+0.7}$ & $-8.9_{-0.9}^{+0.9}$ & $0.3 \pm 0.1$ & $0.3 / 5$ \\
GRB 081109 & 0.979 & $-21.27 \pm 0.09$ & $0.24_{-0.11}^{+0.22}$ & $0.24_{-0.04}^{+0.06}$ & $9.82_{-0.09}^{+0.09}$ & $1.5_{-0.2}^{+0.2}$ & $-8.4_{-0.3}^{+0.3}$ & $0.9 \pm 0.1$ & $11.2 / 14$ \\
GRB 090926B & 1.24 & $-21.5 \pm 0.1$ & $0.14_{-0.09}^{+0.60}$ & $0.35_{-0.05}^{+0.08}$ & $10.1_{-0.5}^{+0.4}$ & $1.9_{-0.5}^{+0.4}$ & $-8.1_{-0.9}^{+0.6}$ & $0.9 \pm 0.1$ & $7.3 / 9$ \\
GRB 100621A & 0.542 & $-20.68 \pm 0.08$ & $0.05_{-0.03}^{+0.07}$ & $0.14_{-0.04}^{+0.03}$ & $8.98_{-0.10}^{+0.14}$ & $1.13_{-0.20}^{+0.15}$ & $-7.9_{-0.3}^{+0.2}$ & $0.6 \pm 0.1$ & $16.5 / 14$ \\
\hline
\end{tabular}

\subsubsection{The host of GRB 080605}

The host of GRB 080605 at $z=1.640$ was discovered in late GROND follow-up observations of the burst field 22 days after the GRB trigger. There is evidence of a $2175 \AA$ dust feature in the SED and spectrum with significant $A_{\mathrm{V}}$ in the range of 0.5-1.3 mag (Greiner et al. 2011; Zafar et al. 2011). The host is bright ( $\left.r^{\prime} \sim 22.8 \mathrm{mag}\right)$, and blue with a flat $g^{\prime}-z^{\prime}$ color, and $(R-K)_{\mathrm{AB}} \sim 0.5 \mathrm{mag}$ as estimated from the best-fit galaxy model (Fig. 8). The SED fit further yields $M_{B}=-22.6 \pm 0.2 \mathrm{mag}$, which is $\sim 2.1 L^{*}$ at $z \sim 1.5$, and $\log \left(M_{*}\left[M_{\odot}\right]\right)=9.6_{-0.2}^{+0.3}$. The dominant stellar population of the host is young $\left(\tau=0.06_{-0.03}^{+0.18} \mathrm{Gyr}\right)$, and there is no evidence for reddening at the $2 \sigma$ level $\left(E_{B-V}^{\prime} \lesssim\right.$ $0.22 \mathrm{mag}$ ). The host galaxy is vigorously star-forming with a SFR of $40_{-20}^{+40} M_{\odot} / \mathrm{yr}$ and a SSFR of $\sim 10 \mathrm{Gyr}^{-1}$.

\subsubsection{The host of GRB 080607}

The afterglow of GRB 080607 is heavily reddened $\left(A_{\mathrm{V}} \sim\right.$ $3 \mathrm{mag}$ ), has a modest $2175 \AA$ dust feature, and is characterized by a strong neutral hydrogen absorber of roughly solar metallicity and molecular gas (Prochaska et al. 2009; Perley et al. 2011b). Data from Chen et al. (2011) show a faint and very red host with $R \sim 27 \mathrm{mag}$ and a synthetic color of $(R-K)_{\mathrm{AB}} \sim 3 \mathrm{mag}$ (Fig. 8). The host is well-described with an extinguished galaxy template $\left(A_{\mathrm{V}}^{\prime} \sim 1-2 \mathrm{mag}\right)$, and physical parameters of $M_{B}=-21.1 \pm 0.3 \mathrm{mag}$, which is $\sim 0.3 L^{*}$ at $z \sim 3, \log \left(M_{*}\left[M_{\odot}\right]\right)=9.9_{-0.6}^{+0.4}$, an extinction-corrected SFR of $40_{-26}^{+60} M_{\odot} / \mathrm{yr}$, and SSFR of $\sim 8 \mathrm{Gyr}^{-1}$. These values are in good agreement with previously published ones (Chen et al. 2011).

\subsubsection{The host of GRB 080805}

GRB 080805 had a very red afterglow, where both an SED and spectral analysis inferred a large dust column $\left(A_{\mathrm{V}} \sim\right.$ $1.0-1.5 \mathrm{mag}$ ), and evidence of a $2175 \AA$ dust feature (Fynbo et al. 2009a; Greiner et al. 2011; Zafar et al. 2011). Its host was discovered in late EFOSC/HAWK-I imaging in five filters (VRiJK, see Fig. 8), is relatively bright $(R \sim 25.5 \mathrm{mag})$ and red $\left((R-K)_{\mathrm{AB}}=2.5 \mathrm{mag}\right)$, with best-fit physical parameters of $M_{B}=-20.4 \pm 0.2 \operatorname{mag}\left(\sim 0.3 L^{*}\right.$ at $\left.z \sim 1.5\right)$ and $\log \left(M_{*}\left[M_{\odot}\right]\right)=$ $9.7_{-0.2}^{+0.2}$. The remaining galaxy properties are poorly constrained by the available data, yielding a limit to the galaxy reddening of $E_{B-V}^{\prime} \lesssim 0.7 \mathrm{mag}$, an age of the stellar population of $0.5_{-0.4}^{+0.8} \mathrm{Gyr}$, a SFR of $6_{-5}^{+25} M_{\odot} / \mathrm{yr}$, and a SSFR of $\sim 1 \mathrm{Gyr}^{-1}$. 


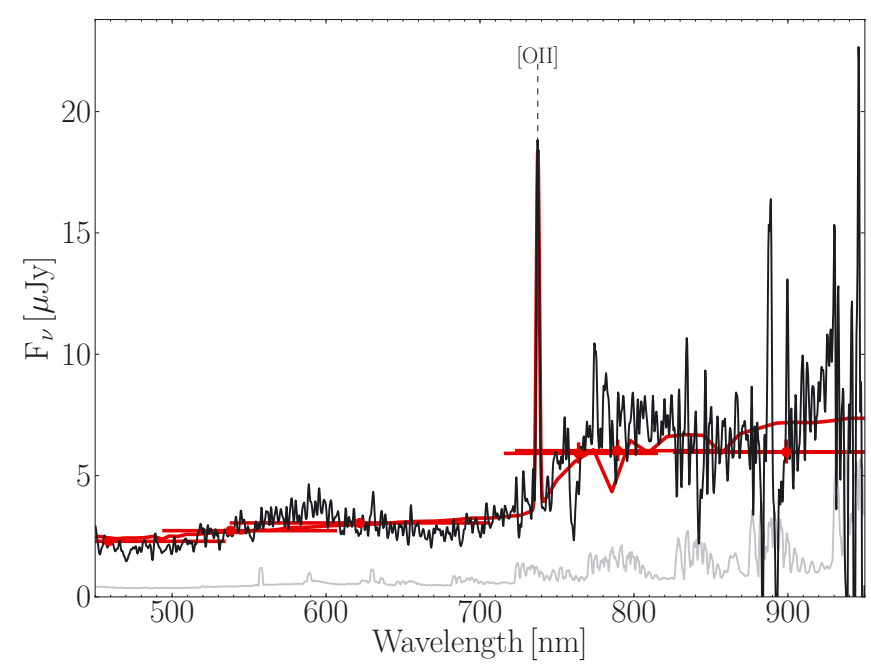

Fig. 9. Wavelength and flux-calibrated FORS2 $300 \mathrm{~V}$ spectrum of the host of GRB 081109 in black. The thin grey line shows the sky spectrum. The red line is the best-fit galaxy model obtained from the available photometry and red points are the photometric measurements.

At a distance of $2.5^{\prime \prime}$ north-east of the afterglow/host position, there is another $R \sim 25 \mathrm{mag}$, and even redder $\left((R-K)_{\mathrm{AB}} \sim\right.$ 4 mag) galaxy, which is a plausible candidate to be the strong Mg II absorbing system at $z=1.20$ reported in Fynbo et al. (2009a).

\subsubsection{The host of GRB 081109}

GRB 081109 is the only burst in the sample where no spectroscopic redshift was available in the literature. However, the host is bright $\left(r^{\prime} \sim 22.7 \mathrm{mag}\right)$, moderately red $\left((R-K)_{\mathrm{AB}}=1.6 \mathrm{mag}\right)$ and our host spectrum (see Fig. 9) reveals a single emission line above a well-detected continuum. Within the wavelength coverage of the spectrum $(\sim 370-950 \mathrm{~nm})$, this emission line is interpreted as [OII][ $\lambda 3727]$ at $z=0.9787 \pm 0.0005$. If it were any of the other prominent nebular lines $(\mathrm{H} \beta$, [OIII], $\mathrm{H} \alpha)$, we would have expected to detect [OII][ 23727$]$ in the spectrum as well. At this redshift, there is also spectroscopic evidence of a Balmer break (see Fig. 9).

The host SED is shown in Fig. 8 and well fit with a young $\left(\tau=0.18_{-0.07}^{+0.19} \mathrm{Gyr}\right)$ and reddened $\left(A_{\mathrm{V}}^{\prime}=1.0 \pm 0.2 \mathrm{mag}\right)$ stellar population. The absolute magnitude of $M_{B}=-21.27 \pm$ $0.09 \mathrm{mag}$ corresponds to $\sim 0.9 L^{*}$ at $z \sim 1$. The stellar mass obtained from the SPS fit is $\log \left(M_{*}\left[M_{\odot}\right]\right)=9.80_{-0.09}^{+0.09}$, with a SFR of $33_{-13}^{+19} M_{\odot} / y r$, which together yields a SSFR of $\sim 5 \mathrm{Gyr}^{-1}$. The emission-line flux of [OII][23727] is $(1.8 \pm$ $0.2) \times 10^{-16} \mathrm{erg} / \mathrm{cm}^{2} / \mathrm{s}$, which includes a systematic error contribution from the absolute flux normalization. This corresponds to a dust un-corrected star-formation rate of $13 \pm 4 M_{\odot} / \mathrm{yr}$ (Kennicutt 1998), or $48_{-16}^{+18} M_{\odot} / \mathrm{yr}$ when using the upper $A_{V}^{\prime}$ measurement, which is in good agreement with the SFR from the UV continuum.

\subsubsection{The host of GRB 090926B}

Promptly after the trigger, Fynbo et al. (2009b) reported the detection of the host galaxy of GRB 090926B based on a prominent [OII] emission line in a VLT/FORS spectrum. The host is also imaged in later GROND and NTT observations with a brightness of $r^{\prime} \sim 23.0 \mathrm{mag}$, and a mildly red color $\left((R-K)_{\mathrm{AB}} \sim\right.$ $1.5 \mathrm{mag})$ as shown in Fig. 8. The host SED is well fit with an extinguished host model $\left(A_{\mathrm{V}}^{\prime}=1.4_{-0.2}^{+0.3} \mathrm{mag}\right)$ with $M_{B}=-21.5 \pm 0.2$ $\left(\sim 0.9 L^{*}\right.$ at $\left.z \sim 1.3\right)$ and $\log \left(M_{*}\left[M_{\odot}\right]\right)=10.1_{-0.5}^{+0.6}$. The SFR based on the extinction-corrected UV flux is $80_{-50}^{+110} M_{\odot} / \mathrm{yr}$ which is among the highest ever measured from optical data, but is only poorly constrained given the uncertainty in the dust extinction properties. The constraints on the age of the stellar population and SSFR are weak, with values of $\tau=0.14_{-0.09}^{+0.60} \mathrm{Gyr}$ and the SSFR of $\sim 7 \mathrm{Gyr}^{-1}$, respectively.

\subsubsection{The host of GRB $100621 \mathrm{~A}$}

The host of GRB 100621A was reported soon after the trigger as a DSS2 source, providing a constant contribution to the afterglow in the UV/blue light curve (Updike et al. 2010; Oates \& Ukwatta 2010). The redshift of $z=0.542$ of GRB 100621A is based on emission lines from a bright host (Milvang-Jensen et al. 2010). The SED of the $r^{\prime} \sim 21.5$ mag host is well-sampled from the UV to the NIR and shown in Fig. 8. In strong contrast to the extremely red afterglow $(R-K)_{\mathrm{AB}} \sim 5.8 \mathrm{mag}$, the host is blue with an inferred color of $(R-K)_{\mathrm{AB}} \sim 0.3$ mag and $(\text { uvw } 2-K)_{\mathrm{AB}} \sim 0.9 \mathrm{mag}$. The SPS host fit returns an intrinsic extinction of $A_{\mathrm{V}}^{\prime}=0.6_{-0.2}^{+0.1}$ mag for a very young stellar population of age $\tau=0.05_{-0.03}^{+0.07} \mathrm{Gyr}$. This host has the lowest stellar mass in the presented sample with $\log \left(M_{*}\left[M_{\odot}\right]\right)=8.98_{-0.10}^{+0.14}$, and an absolute magnitude of $M_{B}=-20.68 \pm 0.08 \mathrm{mag}$, which is $\sim 0.6 L^{*}$ at $z \sim 0.5$. The SFR and SSFR are $13_{-5}^{+6} M_{\odot} / \mathrm{yr}$ and $\sim 14 \mathrm{Gyr}^{-1}$, respectively.

\section{Discussion}

\subsection{Dust reddening in GRB afterglow SEDs}

The visual extinction measured from X-ray-to-NIR SED-fitting towards GRBs $070306\left(A_{\mathrm{V}}^{\mathrm{GRB}}=5.5_{-1.0}^{+1.2} \mathrm{mag}\right), 081109\left(A_{\mathrm{V}}^{\mathrm{GRB}}=\right.$ $\left.3.4_{-0.3}^{+0.4} \mathrm{mag}\right)$, and $100621 \mathrm{~A}\left(A_{\mathrm{V}}^{\mathrm{GRB}}=3.8 \pm 0.2 \mathrm{mag}\right)$ are among the largest ever derived from optical/NIR data for GRB afterglows (e.g., Savaglio et al. 2003; Kann et al. 2006; Greiner et al. 2011). The dust properties inferred from afterglow measurements are well-represented with local models in the rest-frame $300-1100 \mathrm{~nm}$, and at the resolution of broad-band imaging (see also e.g., Fynbo et al. 2001; Watson et al. 2006; Kann et al. 2006; Schady et al. 2007; Starling et al. 2007; Schady et al. 2011b), while noteworthy exceptions exist in the literature (e.g., Savaglio \& Fall 2004; Perley et al. 2010b; Clemens et al. 2011). The good fit provided by local dust-extinction laws suggests an abundance of small dust grains comparable to that of the MW/LMC or SMC. There is hence no direct evidence that the dust towards these GRBs through their hosts is different than observed along local sight-lines. A different dust-grain size distribution would have been expected if the dust were located in the immediate vicinity $\left(R \lesssim 10^{19} \mathrm{~cm}\right)$ of the GRB and shaped through its intense radiation, i.e., through dust destruction (Waxman \& Draine 2000; Fruchter et al. 2001; Draine \& Hao 2002). In addition, the metal-to-dust ratios for these afterglows are only a few times the Galactic value of $N_{\mathrm{H}} / A_{\mathrm{V}} \approx 2 \times 10^{21} \mathrm{~cm}^{-2} / \mathrm{mag}$ (Predehl \& Schmitt 1995). For un-extinguished GRB sight-lines this ratio is generally found to be a factor of 10-100 times larger than those of the Magellanic Clouds or Milky Way (e.g., Galama \& Wijers 2001; Stratta et al. 2004). This is indicative of a dependence of the metal-to-dust ratio on the amount of visual extinction. We return to this issue in Sect. 6.4. 


\subsection{The hosts of the dustiest afterglows}

The general properties of the selected GRB host galaxies are diverse. They have $(R-K)_{\mathrm{AB}}$ colors ranging from flat and blue $(R-K)_{\mathrm{AB}} \sim 0$ mag to extremely red $(R-K)_{\mathrm{AB}} \sim 3$ mag with an average color of $\left\langle(R-K)_{\mathrm{AB}}\right\rangle=1.6 \mathrm{mag}$, and host extinction values between $A_{\mathrm{V}}^{\prime} \sim 0$ mag and $A_{\mathrm{V}}^{\prime} \sim 2$ mag. In addition, their stellar mass and absolute magnitude distributions are broad, with values between $\log \left(M_{*}\left[M_{\odot}\right]\right)=9.0$ to $\log \left(M_{*}\left[M_{\odot}\right]\right)=10.4$ $\left(\left\langle\log \left(M_{*}\left[M_{\odot}\right]\right)\right\rangle=9.8 \pm 0.4\right)$ and $M_{B}$ between $-20.3 \mathrm{mag}$ and $-22.6 \mathrm{mag}\left(\left\langle M_{B}\right\rangle=-21.3 \pm 0.6 \mathrm{mag}\right)$. These absolute brightnesses correspond to a range between several tenths and a few $L^{*}\left(\langle L\rangle=0.9 L^{*}\right)$. The average SFR and SSFR are about $30 M_{\odot} / \mathrm{yr}$ and $\langle\log S S F R$ [yr] $\rangle \sim-8.3$, respectively. The average growth time is $\sim 0.2 \mathrm{Gyr}$, which illustrates that not only opticallyselected hosts, but also the hosts of highly-reddened afterglows are very efficient in producing stars. A rough estimate of the metallicity of the hosts can be obtained if these GRB hosts follow the fundamental plane as defined for nearby SDSS galaxies (Mannucci et al. 2010a). With a given stellar mass and SFR, the host galaxies in this sample are expected to have metallicities in a range between $12+\log (\mathrm{O} / \mathrm{H}) \sim 8.2$ and $12+\log (\mathrm{O} / \mathrm{H}) \sim 8.9$, with an average of $12+\log (\mathrm{O} / \mathrm{H}) \sim 8.6$. We caution that the SFRs were derived using the rest-frame UV flux, which is quite sensitive to the dust extinction properties.

Although not well-constrained in all cases, the average luminosity-weighted effective reddening inferred from host photometry is typically smaller or equal to that measured from afterglow observations. This is not a particularly surprising result, given that the sample selection was based on high visual extinctions of the afterglow SEDs in the first place. It directly indicates some variation in the dust distribution of the hosts, which again is not a surprising result, given the differences in extinction properties along different sight-lines through the diffuse ISM to giant molecular clouds in the Local Group (e.g., Gordon et al. 2003; Fitzpatrick 2004), and the geometrical differences between a single sight-line and an extended distribution of star-light and dust (e.g., Gordon et al. 1997; Silva et al. 1998).

One intriguing case is the host of GRB 100621A. Although having one of the most extinguished afterglows ever detected (even in the presented sample), its host shows very blue colors, and is one of the youngest and the least massive galaxy in this work. This particular example provides evidence of a patchy dust component where the geometry of the dust distribution, and not the properties of the host galaxy, makes the specific GRB sightline dust-enriched.

\subsection{Comparison to previous GRB host samples}

One key result of this study is the success rate of the discovery of the selected hosts. Out of eight hosts, which were selected based on their afterglow properties (hence a selection independent of host properties, in particular galaxy brightness), all are luminous enough to be detected in optical ground-based imaging. This fraction is significantly larger than expected from a host sample based on XRT detections (Fynbo et al. 2008; Malesani et al. 2009). The effect of a higher detection rate is even stronger in the NIR: seven out of eight sources are detectable in the $K$-band, while this fraction is only $\sim 35 \%$ for the general host population of Swift/GRBs (Malesani et al. 2009). This is partially the result of the lower average redshift of the selected hosts $\left(\left\langle z_{A_{\mathrm{V}}}\right\rangle=1.5\right)$ relative to all Swift GRBs with $\left\langle z_{\text {Swift }}\right\rangle \sim 1.9$.

The lower redshift is however not the only reason for the high detection rate. The selected hosts are on average redder and, as
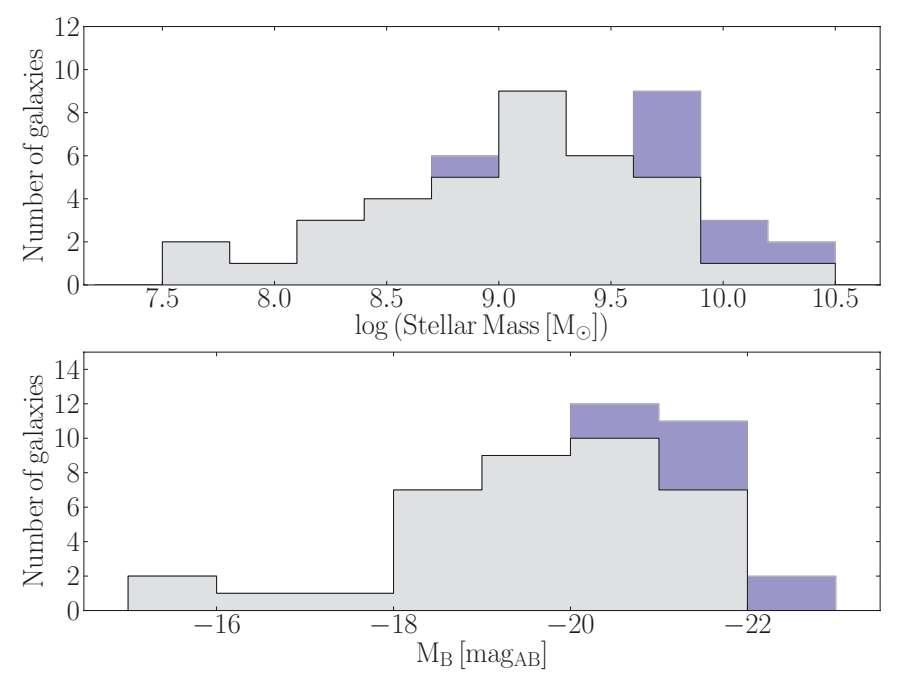

Fig. 10. Distribution of stellar masses and luminosities of the hosts of highly extinguished afterglows (blue) and the host sample from SGL09 (grey).

shown in Fig. 10, have typically higher luminosities and stellar masses than the (sensitivity-limited) SGL09 sample, which has $\left\langle(R-K)_{\mathrm{AB}}\right\rangle=0.8 \mathrm{mag},\left\langle M_{B}\right\rangle=-19.6 \pm 1.5 \mathrm{mag}$, and $\left\langle\log M_{*}\left[M_{\odot}\right]\right\rangle=9.1 \pm 0.6$. A two-sample K-S test returns $p$ values of 0.002 for the stellar mass, and 0.006 for the absolute magnitude distributions respectively, which is tentative evidence that both distributions are not drawn from the same parent sample. However, given the small sample size of only eight high- $A_{\mathrm{V}}$ events, larger samples are required to statistically establish the existence of a difference at higher significance. Both distributions are of course drawn from the same physical parent sample (GRB hosts), indicating that the different selection criteria probe different host properties.

A possible explanation of the different host properties would be the now on-average higher redshift than the SGL09 sample, where star formation was driven by more massive galaxies than in the more nearby Universe (e.g., Cowie et al. 1996; Hopkins 2004). To test this hypothesis, we selected a sub-sample from SGL09 with a median redshift comparable to the hosts in this work. This essentially removes all $z<1$ SGL09 hosts and leaves only 13 events for comparison (see histograms in Fig. 11). Despite the small number statistics, the $M_{*}$ and $M_{B}$ values are again placed at the high-mass and high-luminosity end of their respective distribution, and a K-S test also provides marginal evidence of a difference ( $p$-values of 0.001 and 0.034 for the masses and absolute magnitudes).

We conclude that by selecting extinguished afterglows we very likely probe a more luminous, massive, and chemically evolved population of GRB hosts.

As it is clear that these were largely missing from previous samples, there is a selection bias and the GRB host population is missing most of its massive, evolved and metal-rich members. As a direct consequence, GRB hosts trace the global SFR more reliably than indicated in studies that are based on host samples of optically selected GRB afterglows, and the apparent deficiency of high-mass host galaxies is at least partially a selection effect.

Similar conclusions apply to all galaxies hosting afterglows that have a significant $2175 \AA$ dust feature in their SED. Four out of five currently known afterglows are within the presented sample, which implies that there is a direct connection between 
T. Krühler et al.: The SEDs and host galaxies of the dustiest GRB afterglows
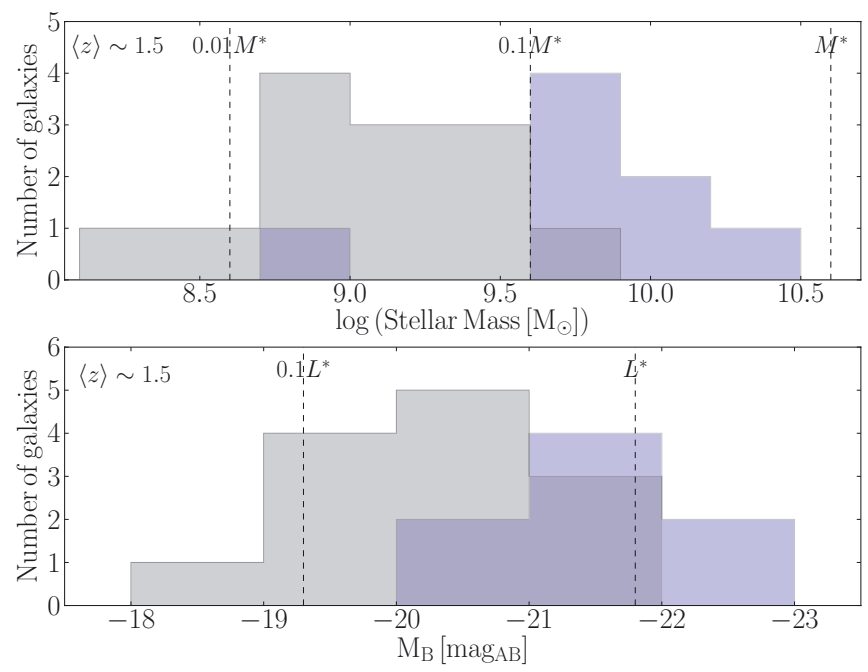

Fig. 11. Distribution of stellar masses and luminosities of the hosts of highly extinguished afterglows (blue) and a subsample of SGL09 (grey) with $\langle z\rangle \sim 1.5$.

large dust columns and the presence of the UV bump (see e.g., Greiner et al. 2011; Zafar et al. 2011). Their, on average, more massive and luminous hosts suggest a qualitative relation between the stellar mass of a galaxy and the presence of a $2175 \AA$ feature, where the latter is only present in fairly massive and metal-enriched galaxies (see also e.g., Noll \& Pierini 2005). Conversely, a strong $2175 \AA$ feature in an afterglow SED is also very likely a good proxy for the stellar mass and luminosity of the GRB host.

\subsection{Metal-to-dust ratios in context}

The ratio of the line-of-sight extinction to the total metal column for GRB afterglows has been investigated in a number of papers (e.g., Galama \& Wijers 2001; Stratta et al. 2004; Savaglio \& Fall 2004; Kann et al. 2006; Schady et al. 2007, 2010; Greiner et al. 2011; Zafar et al. 2011), where ratios typically much higher than the ones observed in the Local Group were derived. Measurements for different Galactic sight-lines (e.g., Predehl \& Schmitt 1995; Güver \& Özel 2009) show an almost universal value of around $N_{\mathrm{H}} / A_{\mathrm{V}} \approx 2 \times 10^{21} \mathrm{~cm}^{-2} / \mathrm{mag}$, while the matter probed by afterglows can yield metal-to-dust ratios up to and sometimes even above 100-times higher (e.g., Watson et al. 2007; Rau et al. 2010).

\subsubsection{An anti-correlation between metal-to-dust ratio and sight-line extinction}

Figure 12 shows the $N_{\mathrm{H}, \mathrm{X}} / A_{\mathrm{V}}^{\mathrm{GRB}}$ ratio for a large number of afterglows and illustrates its dependence on the sight-line dust extinction. With the afterglows in this work, there is now for the first time reasonable coverage in the $A_{\mathrm{V}}^{\mathrm{GRB}} \sim 1-5 \mathrm{mag}$ range. Intriguingly, the metal-to-dust ratio is strongly anti-correlated with $A_{\mathrm{V}}^{\mathrm{GRB}}$, confirming the tentative trend reported by Perley et al. (2009). A Spearman rank-order correlation analysis for the combined sample in Figure 12 returns a correlation coefficient $\rho=-0.63$, with a two-tailed $p$-value of $3 \times 10^{-7}$, in strong contrast to a constant, universal $N_{\mathrm{H}, \mathrm{X}} / A_{\mathrm{V}}^{\mathrm{GRB}}$ ratio.

There are two straightforward ways to reconcile this result. One is a dependence of the metal-to-dust ratio on the specific environment such that evolved and dust-enriched hosts are more

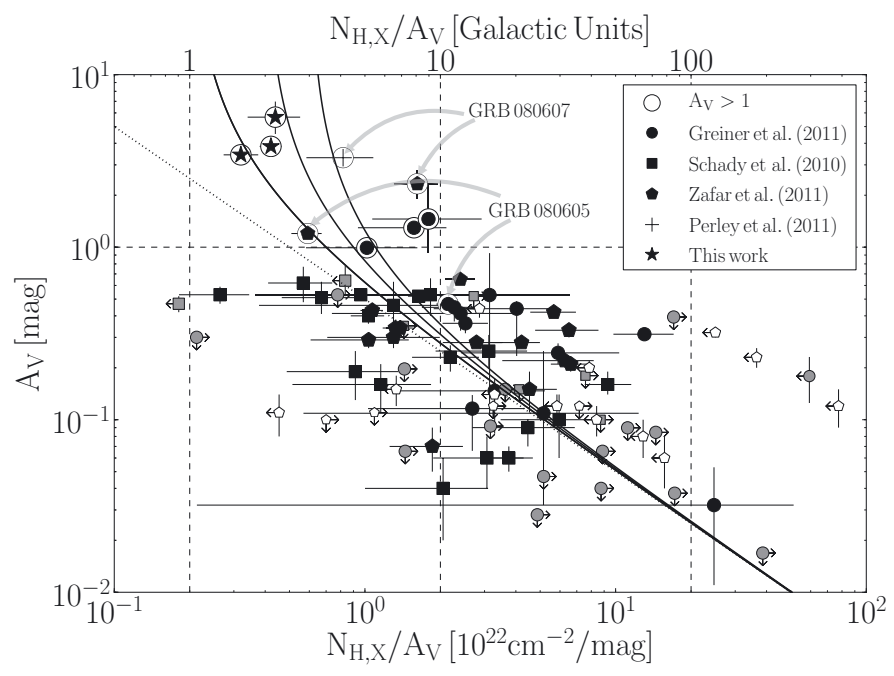

Fig. 12. Metal-to-dust ratio versus sight-line extinction for GRB afterglows. The horizontal dashed lines marks the selection criterion for GRBs to enter this sample. Vertical dashed lines illustrate metal-to-dust ratios of 1,10 or 100 times the Galactic value. Solid lines show the toy model of two physically independent absorbers, where one is fully devoid of dust with a H-equivalent metal column of $N_{\mathrm{H}, \mathrm{X}}=10^{21.7} \mathrm{~cm}^{-2}$ and represented by the dotted line, while the other is neutral and has metal-to-dust ratios of 1, 2 or 3 times the Local Group value. Two individual cases (GRBs 080605 and 080607) illustrate the scatter between the analysis of different data sets.

efficient in forming dust out of their metals (and in fact we do observe on average higher stellar masses for the hosts of high $A_{\mathrm{V}}^{\mathrm{GRB}}$ afterglows). The other is the presence of two physically independent absorbers, where the first dominates the total metal column, and the second the visual extinction measurements. This trivially produces a non-correlation between $N_{\mathrm{H}, \mathrm{X}}$ and $A_{\mathrm{V}}^{\mathrm{GRB}}$, and consequently an $N_{\mathrm{H}, \mathrm{X}} / A_{\mathrm{V}}^{\mathrm{GRB}}$ to $A_{\mathrm{V}}^{\mathrm{GRB}}$ anti-correlation.

Some outliers and scatter of the metal-to-dust anticorrelation (Fig. 12) might be caused by difficulties in measuring the respective physical parameters. This is also illustrated by the example of two individual events (GRBs 080605 and 080607), where different values have been published in the literature. Assumptions about the continuum emission, the extinction law, and the total-to-selective reddening $R_{\mathrm{V}}$ can affect the $A_{\mathrm{V}}^{\mathrm{GRB}}$ measurement. In addition, there is the possibility that the cooling break is located close to or within the range of the UV/optical/NIR measurements. In a standard analysis, the introduced curvature caused by the spectral break is then interpreted as a larger dust column (Krühler et al. 2011). The $N_{\mathrm{H}, \mathrm{X}}$ measurements are also prone to errors: spectral variation intrinsic to the afterglow can lead to incorrect estimates of the soft X-ray absorption (e.g., Butler \& Kocevski 2007).

\subsubsection{Metal-to-dust ratio compared to host mass}

As shown in Sect. 6.2, the hosts of dusty afterglows are on average more massive and luminous than their non-extinguished counterparts, but there is a broad range of galaxy properties and the only common feature between all afterglows/hosts in this work is hence the dusty line of sight. In particular, if the environment were responsible for the observed $N_{\mathrm{H}, \mathrm{X}} / A_{\mathrm{V}}^{\mathrm{GRB}}$ to visual extinction anti-correlation, we would expect the metal-to-dust ratio for GRB 100621A to be comparable to the bulk of optically bright afterglows. It is, however, one of the lowest ever observed for GRB afterglows and a factor of five lower than the median 


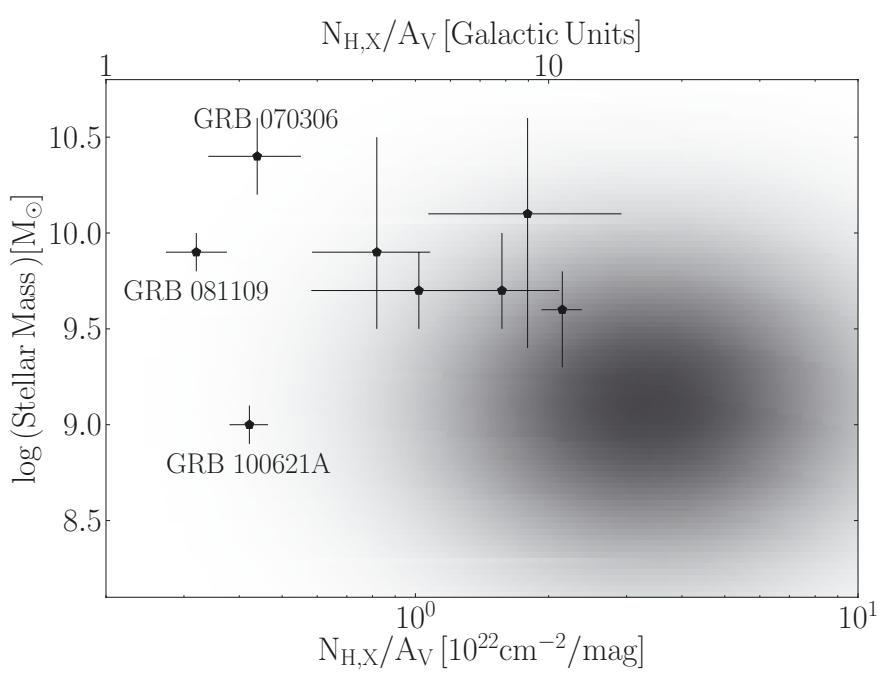

Fig. 13. Total metal-to-dust ratios for GRB afterglows versus stellar mass of their host galaxies. Black data are galaxies hosting a highlyextinguished afterglow using the first values of Table 5, where the three hosts with the highest $A_{\mathrm{V}}^{\mathrm{GRB}}$ are labeled. The shaded area indicates the probability distribution for optically-selected GRBs, represented by a log-normal distribution of $N_{\mathrm{H}, \mathrm{X}} / A_{\mathrm{V}}^{\mathrm{GRB}}$ based on Schady et al. (2010) and a Gaussian distribution in $\log \left(M_{*}\left[M_{\odot}\right]\right)$ based on SGL09.

for afterglows with hosts of similar mass (see Fig. 13). Although we note that number counts are still too low to derive strong constraints with high statistical significance, this suggests that the specific host environment is not responsible for the observed dependence of the metal-to-dust ratio on the visual extinction.

\subsubsection{A system of two absorbers}

In the second scenario, two largely independent (one neutral, host-galaxy related, one ionized, circumburst specific) columns of material contribute to the observed absorption, denoted as $N_{\mathrm{H} \text {,neutral }}, N_{\mathrm{H} \text {,ion }}$ in the following. $N_{\mathrm{H}, \mathrm{X}}$ measures the sum of both, whereas the $A_{\mathrm{V}}^{\mathrm{GRB}}$ column would only be associated with the $N_{\mathrm{H}, \text { neutral }}$ absorber. This circumburst environment is not unexpected: the intense afterglow radiation should not only photoionize the vicinity of the burst, but also destroy the associated dust in large amounts (Waxman \& Draine 2000; Draine \& Hao 2002; Perna et al. 2003), albeit with different effective radii.

For $A_{\mathrm{V}}^{\mathrm{GRB}} \sim 0.1 \mathrm{mag}$ sight-lines, $N_{\mathrm{H} \text {,neutral }} \ll N_{\mathrm{H} \text {,ion }}$ directly results in a large metal-to-dust ratio. With an increasing $A_{\mathrm{V}}^{\mathrm{GRB}}$ column of around $1 \mathrm{mag}$, the $N_{\mathrm{H} \text {,neutral }}$ absorber contributes significantly to the total metal column $\left(N_{\mathrm{H} \text {,neutral }} \approx N_{\mathrm{H} \text {,ion }}\right)$ : this is illustrated in Fig. 12 by the solid lines, where the $N_{\mathrm{H}, \mathrm{X}} / A_{\mathrm{V}}^{\mathrm{GRB}}$ ratio asymptotically reaches one, two or three times the Local Group value. Even larger $A_{\mathrm{V}}^{\mathrm{GRB}}$ columns than present in this work can test this hypothesis. For an $A_{\mathrm{V}}^{\mathrm{GRB}} \gtrsim 10$ mag sight-line, for example, $N_{\mathrm{H} \text {,neutral }}$ is much larger than $N_{\mathrm{H} \text {,ion }}$ and the expected metalto-dust ratio would be comparable to its intrinsic value, and to the Local Group value (if universal).

For the bulk of standard, un- or mildly extinguished afterglows, the large column of ionized metals with an equivalent $N_{\mathrm{H}, \text { ion }} \sim 10^{21-22} \mathrm{~cm}^{-2}$ in the circumburst material dominates the total $N_{\mathrm{H}, \mathrm{X}}$ measurement, whereas the visual extinction is very likely caused by dust further out, either in the diffuse ISM, or localized in interstellar clouds (see also Sect. 6.5). Hence, for optically bright GRBs the total metal absorption as probed by the soft X-ray absorption is neither a good measure nor a direct tracer of the dust extinction along the line of sight. In other words, an $N_{\mathrm{H}, \mathrm{X}}$ column even as large as $10^{22} \mathrm{~cm}^{-2}$ does not necessarily imply a significant visual extinction (see also, e.g., Galama \& Wijers 2001; Kann et al. 2006; Schady et al. 2007).

We thus conclude that the anti-correlation between the metal-to-dust ratio and the sight-line extinction indicates the presence of two absorbing systems, which are to first order physically independent. One of them is dusty, the other ionized and dust-free in most of the cases, where the former is probed by the optical/NIR data and the latter typically dominates the $N_{\mathrm{H}, \mathrm{X}}$ measurement.

\subsection{Location and geometry of the absorbing dust column}

A natural question about the nature of the absorbing dust, gas, and metal columns detected in the afterglow SEDs and spectra is their locations, and whether they are directly related to the burst environment. A number of previous studies have already revealed some clues about the geometry of the absorbing matter, which have been derived quite exclusively from sight-lines of low total dust content: the distance of the cold-neutral material, linked to the DLA and the low-ionization metal absorption lines has been constrained to be between a few hundreds of pc and a few kpc (Prochaska et al. 2006; Vreeswijk et al. 2007). In contrast, the high metal column densities as derived from soft X-ray absorption were associated with a fully ionized circumburst medium up to a few to several tens of pc (Watson et al. 2007; Schady et al. 2011a). For bursts with largely unextinguished afterglows, most of the metals along the sight-line are typically in a highly ionized state and only $\lesssim 10 \%$ of the absorbing gas is neutral (Schady et al. 2011a). In addition, there is no statistically significant correlation between the soft X-ray absorption and neither the dust column as inferred from optical/NIR data nor the metallicity of the neutral material (e.g., Schady et al. 2007; Zafar et al. 2011), nor the darkness of a GRB (Campana et al. 2010; see however Fynbo et al. 2009a). There is however a trend of higher visual extinctions with larger neutral metal columns and an anti-correlation between gas-to-dust ratio and metallicity (Zafar et al. 2011). Dark bursts also have stronger neutral metal absorption lines in their optical spectrum (Christensen et al. 2011).

The dustiest afterglows and their hosts provide two additional pieces of information. Firstly, there is the previously discussed anti-correlation between metal-to-dust ratio and sightline extinction, albeit with a large scatter for individual events. And secondly, their hosts are on average redder, more luminous and massive, and supposedly also more evolved, dust- and metal-rich than their low- $A_{\mathrm{V}}^{\mathrm{GRB}}$ counterparts (e.g., Kobulnicky \& Kewley 2004; Savaglio et al. 2005). There is hence a relation between the dust along the sight-line towards the GRB and physical host properties. This relation is expected if the dust probed by the afterglow is located at large enough distances to be fairly representative of the size of the host galaxy, its global dust enrichment, and its chemical state. In contrast, dust in the surrounding environment of a GRB, even if present and able to survive the intense afterglow or progenitor radiation, should instead be related to the very specific details of its circumburst environment or the GRB's birth cloud.

An overlap with optically selected hosts clearly exist: very young, blue, and low-mass galaxies possess sight-lines through dusty regions as demonstrated through the afterglows and hosts of, e.g., GRBs 080605 and 100621A. For this kind of events, the data are clearly not consistent with a uniform dust-shield. In a few cases, the observations instead indicate a patchy dust 
distribution where the dust is located in clumps of small enough covering factor and large enough extinction to be negligible in the integrated host-light distribution. The variations in the visual extinction in some cases are therefore a geometrical effect (see also, e.g., Berger et al. 2003; Perley et al. 2009).

This raises questions about the validity of star-formation estimates obtained for young and blue galaxies. Afterglow observations show that extinguished star-formation takes place even in apparently unextinguished galaxies. Here, either the covering factor of the dusty clump is just too small to remove significantly from the host light, or the clump completely absorbs all UV light from the star-forming region, thus having a negligible effect on the host galaxy colors. In the latter case, the UV-derived SFRs would strictly represent lower limits to the star-formation in these galaxies. Far-infrared, sub-mm or radio observations with Herschel, ALMA or the VLA, respectively, would enable a measurement of the galaxies global SFR, and its dust mass and temperature and help to clarify this issue.

A coherent picture of highly extinguished afterglows in combination with their diverse, but on average redder and more massive hosts could be obtained within a complex dust distribution made out of several constituents related to extinction in the diffuse ISM, extended interstellar clouds, or localized in fairly compact and dense regions such as giant molecular clouds.

The dust column density is hence very likely not directly located in the GRB environment but plausibly with the neutral absorber at distances of a few hundred pc to one kpc. We stress, however, that the effective radii of dust destruction and photoionization will shape the detailed gas-to-dust and metal-to-dust ratios, adding further complexity to the absorbing system(s) in front of GRB afterglows. Furthermore, the dust distribution in high- $z$ galaxies might be even more complex because of dusty galaxy outflows, and reflect the absence of a uniform chemical enrichment on scales up to one kpc (e.g., Noll et al. 2009).

\section{Conclusions}

The afterglows of GRBs $081109\left(A_{\mathrm{V}}^{\mathrm{GRB}}=3.4_{-0.3}^{+0.4} \mathrm{mag}\right)$ and $100621 \mathrm{~A}\left(A_{\mathrm{V}}^{\mathrm{GRB}}=3.8 \pm 0.2 \mathrm{mag}\right)$ join the growing sample of highly extinguished events. Their continuum emission is wellconstrained by the combination of X-ray and NIR data, and the optical observations provide a detailed measurement of the dust properties along the sight-lines. While some diversity in their extinction properties, particularly dust abundance, clearly exists, GRBs 081109 and 100621A provide compelling evidence that a highly obscured afterglow is also a highly reddened one, and that extinction laws derived from local sight-lines accurately estimate the dust properties towards even highly extinguished GRBs.

The availability of a large enough sample of coeval afterglows with multi-wavelength data would ideally enable to advance from single sight-line, pencil-beam investigations to a statistically symmetric geometry, where each GRB afterglow represents a different sight-line through its host galaxy. In analogy to the case studied by Witt \& Gordon (1996), this could provide a good description of the structure and evolution of the absorbing medium and help to constrain the opacity and filling factors of the dust geometry and clumps from the distribution of $A_{\mathrm{V}}^{\mathrm{GRB}}$ values in star-forming galaxies out to very high redshift.

The hosts of the dustiest afterglows provide a different picture of GRB host galaxies compared to the hosts of opticallyselected bursts. Although both samples overlap in terms of their properties, the galaxies in this work have typical luminosities of around $L^{*}$ and stellar masses of $M_{*} \sim 10^{10} M_{\odot}$, hence more luminous and massive than the hitherto discovered, on average faint and blue hosts. Although the number counts are still low, this work indicates that a selection based on a large $A_{\mathrm{V}}^{\mathrm{GRB}}$ picks up preferentially the more massive and chemically-evolved GRB hosts, which is in qualitative agreement with observations (Perley et al. 2010a, 2011a; Rossi et al. 2011b), and theoretical expectations (Campisi et al. 2011) for the properties of dark GRB host galaxies.

This suggests that the properties of complete GRB host samples are diverse, and complex selection biases are still present: not only are the very faintest GRB hosts missing because of inherent sensitivity limits, but also some of the brightest, most luminous, and chemically evolved ones, because they have not been localized accurately enough. Fairly large and massive, dusty, and metal-rich galaxies are able to host GRBs, and the trend of low-metallicity GRB hosts is not as significant as claimed in previous studies, and possibly a selection effect of the young galaxy population dominating the global SFR at low- $z$ (e.g., Berger et al. 2007; Mannucci et al. 2011). This has substantial implications for the feasibility of tracing the star-formation history with GRB hosts and also for the progenitor channels of GRB production as a result of their metallicity dependence. In the former case, this work indeed indicates that the deficiency of high-mass GRB host galaxies in previous studies was at least partially due to a selection bias. The latter case, however, depends quite strongly on the assumption that the host-inferred metallicities are representative of the composition of the progenitor star, while different sight-lines through a GRB host can show a dispersion in metallicity of around a factor 100 (Pontzen et al. 2010).

Intriguingly, all GRBs with $A_{\mathrm{V}}^{\mathrm{GRB}} \gtrsim 4$ mag have metal-todust ratios significantly below what is typically measured for GRB afterglows, and more in line with measurements from the Local Group. In addition, there is a strong anti-correlation between the metal-to-dust ratio and the visual extinction along the GRB sight-line. This effect seems independent of the specific host properties and can be interpreted as evidence of two physically independent absorbers: dust-free, ionized metals in the circumburst environment (typically probed by the soft X-ray absorption), and in contrast a dusty absorber further out (probed by reddening measurements in the UV/optical/NIR).

The existence of a dust column independent of the immediate circumburst environment is further supported by the relation between afterglow $A_{\mathrm{V}}^{\mathrm{GRB}}$ and host properties, in particular the on-average higher stellar mass and redder colors. Coupled with the blue and very young hosts of, e.g., GRBs 080605 or 100621A, this provides evidence of a complex dust geometry in star-forming galaxies with different constituents in the diffuse ISM and in localized patches, which are plausibly associated with the cold-neutral absorber detected in rest-frame UV/optical GRB afterglow spectra.

Further advances can now be made by getting direct observational access to more dusty sight-lines including $A_{\mathrm{V}}^{\mathrm{GRB}} \gtrsim$ 10 mag events, including those at higher redshifts. Similar observations of a large enough sample would investigate the dependence of the global dust enrichment on cosmic evolution and vice versa, and constrain the fraction of dust-enshrouded star-formation out to very high redshifts. A sophisticated observational strategy coupled with state-of-the-art instrumentation makes such a challenging study feasible. A rapid response of the order of several minutes by a NIR imager at an $8 \mathrm{~m}$-class telescope would have enabled the detection of the afterglows of GRB $100621 \mathrm{~A}(z \sim 0.5)$ up to $A_{\mathrm{V}}^{\mathrm{GRB}} \approx 30 \mathrm{mag}, \mathrm{GRB} 081109$ 
$(z \sim 1)$ up to $A_{\mathrm{V}}^{\mathrm{GRB}} \approx 20 \mathrm{mag}$, and GRB $070802(z \sim 2.5)$ up to $A_{\mathrm{V}}^{\mathrm{GRB}} \approx 10$ mag, which subsequently could have been followedup using NIR spectroscopy. Once an accurate position as well as detailed information about the GRB sight-line is available, their hosts are readily accessible for multi-wavelength surveys via large ground- and space-based facilities, yielding information about otherwise fully extinguished environments and unprecedented insights into the conditions of star-forming galaxies throughout the Universe.

Acknowledgements. We thank the referee for valuable comments. We also acknowledge helpful comments from S. Shore, R. Salvaterra, M. Michalowski and E. Ramirez-Ruiz. T.K. acknowledges support by the DFG cluster of excellence "Origin and Structure of the Universe" and support by the European Commission under the Marie Curie IEF Programme in FP7. Part of the funding for GROND (both hardware as well as personnel) was generously granted from the LeibnizPrize to Prof. G. Hasinger (DFG grant HA 1850/28-1). The Dark Cosmology Centre is funded by the Danish National Research Foundation. P.S. acknowledges support by DFG grant SA 2001/1-1. S.S. acknowledges support through project M.FE.A.Ext 00003 of the MPG. S.K., D.A.K. and A.N.G. acknowledge support by DFG grant K1 766/16-1. A.Ro. acknowledges support from the BLANCEFLOR Boncompagni-Ludovisi, née Bildt foundation. S.M.B. acknowledges support of a European Union Marie Curie European Reintegration Grant within the 7th Program under contract number PERG04-GA-2008-239176. This work made use of data supplied by the UK Swift Science Data Centre at the University of Leicester.

\section{References}

Abazajian, K. N., Adelman-McCarthy, J. K., Agüeros, M. A., et al. 2009, ApJS, 182,543

Adelberger, K. L., \& Steidel, C. C. 2000, ApJ, 544, 218

Anders, E., \& Grevesse, N. 1989, Geochim. Cosmochim. Acta, 53, 197

Appenzeller, I., Fricke, K., \& Fürtig, W., et al. 1998, The Messenger, 94, 1

Arnaud, K. A. 1996, in Astronomical Data Analysis Software and Systems V, ed. G. H. Jacoby \& J. Barnes, ASP Conf. Ser., 101, 17

Barthelmy, S. D., Barbier, L., Cummings, J., et al. 2007, GCN, 6173

Baumgartner, W. H., Barthelmy, S. D., Cummings, J. R., et al. 2009, GCN, 9939 Beardmore, A. P., Page, K. L., \& Immler, S. 2008, GCN, 8506

Bell, E. F., McIntosh, D. H., Katz, N., \& Weinberg, M. D. 2003, ApJS, 149, 289

Berger, E., Cowie, L. L., Kulkarni, S. R., et al. 2003, ApJ, 588, 99

Berger, E., Fox, D. B., Kulkarni, S. R., Frail, D. A., \& Djorgovski, S. G. 2007, ApJ, 660, 504

Blain, A. W., \& Natarajan, P. 2000, MNRAS, 312, L35

Bloom, J. S., Djorgovski, S. G., Kulkarni, S. R., \& Frail, D. A. 1998, ApJ, 507, L25

Bloom, J. S., Kulkarni, S. R., \& Djorgovski, S. G. 2002, AJ, 123, 1111

Bruzual, G., \& Charlot, S. 2003, MNRAS, 344, 1000

Butler, N. R., \& Kocevski, D. 2007, ApJ, 663, 407

Calzetti, D., Armus, L., Bohlin, R. C., et al. 2000, ApJ, 533, 682

Campana, S., Thöne, C. C., de Ugarte Postigo, A., et al. 2010, MNRAS, 402, 2429

Campisi, M. A., Tapparello, C., Salvaterra, R., Mannucci, F., \& Colpi, M. 2011, MNRAS, submitted [arXiv: 1105.1378]

Castro Cerón, J. M., Michałowski, M. J., Hjorth, J., et al. 2010, ApJ, 721, 1919

Cenko, S. B., Kelemen, J., Harrison, F. A., et al. 2009, ApJ, 693, 1484

Chabrier, G. 2003, PASP, 115, 763

Chabrier, G., Baraffe, I., Allard, F., \& Hauschildt, P. 2000, ApJ, 542, 464

Chapman, S. C., Blain, A. W., Smail, I., \& Ivison, R. J. 2005, ApJ, 622, 772

Chen, H., Perley, D. A., Wilson, C. D., et al. 2010, ApJ, 723, L218

Chen, H., Perley, D. A., Wilson, C. D., et al. 2011, ApJ, 727, L53

Christensen, L., Hjorth, J., \& Gorosabel, J. 2004, A\&A, 425, 913

Christensen, L., Fynbo, J. P. U., Prochaska, J. X., et al. 2011, ApJ, 727, 73

Clemens, C., Kruehler, T., \& Greiner, J. 2008a, GCN, 8515

Clemens, C., Kruehler, T., Greiner, J., \& Szokoly, G. 2008b, GCN, 8510

Clemens, C., Greiner, J., Krühler, T., et al. 2011, A\&A, 529, A110

Cool, R. J., Eisenstein, D. J., Hogg, D. W., et al. 2007, GCN, 6170

Covino, S., D’Avanzo, P., Klotz, A., et al. 2008, MNRAS, 388, 347

Cowie, L. L., Songaila, A., Hu, E. M., \& Cohen, J. G. 1996, AJ, 112, 839

Cucchiara, A., Levan, A. J., Fox, D. B., et al. 2011, ApJ, 736, 7

Cummings, J., Barbier, L., Barthelmy, S. D., et al. 2007, GCN, 6699

Cummings, J., Barthelmy, S. D., Baumgartner, W., et al. 2008, GCN, 7841

D’Avanzo, P., Covino, S., Antonelli, L. A., et al. 2008, GCN, 8501

Draine, B. T., \& Hao, L. 2002, ApJ, 569, 780

Elíasdóttir, Á., Fynbo, J. P. U., Hjorth, J., et al. 2009, ApJ, 697, 1725
Evans, P. A., Beardmore, A. P., Page, K. L., et al. 2007, A\&A, 469, 379 Evans, P. A., Beardmore, A. P., Page, K. L., et al. 2009, MNRAS, 397, 1177 Filgas, R., Krühler, T., Greiner, J., et al. 2011, A\&A, 526, A113

Fitzpatrick, E. L. 2004, in Astrophysics of Dust, ed. A. N. Witt, G. C. Clayton, \& B. T. Draine, ASPC Conf. Ser., 309, 33

Fontana, A., Salimbeni, S., Grazian, A., et al. 2006, A\&A, 459, 745

Fruchter, A., Krolik, J. H., \& Rhoads, J. E. 2001, ApJ, 563, 597

Fruchter, A. S., Levan, A. J., Strolger, L., et al. 2006, Nature, 441, 463

Fryer, C. L., Woosley, S. E., \& Hartmann, D. H. 1999, ApJ, 526, 152

Fryer, C. L., Mazzali, P. A., Prochaska, J., et al. 2007, PASP, 119, 1211

Fynbo, J. P. U., Jensen, B. L., Gorosabel, J., et al. 2001, A\&A, 369, 373

Fynbo, J. P. U., Jakobsson, P., Möller, P., et al. 2003, A\&A, 406, L63

Fynbo, J. P. U., Hjorth, J., Malesani, D., et al. 2008, in The Eleventh Marcel

Grossmann Meeting, ed. H. Kleinert, R. T. Jantzen, \& E. Ruffini, 726

Fynbo, J. P. U., Jakobsson, P., Prochaska, J. X., et al. 2009a, ApJS, 185, 526

Fynbo, J. P. U., Malesani, D., Jakobsson, P., \& D'Elia, V. 2009b, GCN, 9947

Galama, T. J., Vreeswijk, P. M., van Paradijs, J., et al. 1998, Nature, 395, 670

Galama, T. J., \& Wijers, R. A. M. J. 2001, ApJ, 549, L209

Gehrels, N., Chincarini, G., Giommi, P., et al. 2004, ApJ, 611, 1005

Gehrels, N., Ramirez-Ruiz, E., \& Fox, D. B. 2009, ARA\&A, 47, 567

Ghisellini, G., Nardini, M., Ghirlanda, G., \& Celotti, A. 2009, MNRAS, 393, 253

Glazebrook, K., Abraham, R. G., McCarthy, P. J., et al. 2004, Nature, 430, 181

Gordon, K. D., Calzetti, D., \& Witt, A. N. 1997, ApJ, 487, 625

Gordon, K. D., Clayton, G. C., Misselt, K. A., Landolt, A. U., \& Wolff, M. J. 2003, ApJ, 594, 279

Granot, J., \& Sari, R. 2002, ApJ, 568, 820

Greiner, J., Bornemann, W., Clemens, C., et al. 2008, PASP, 120, 405

Greiner, J., Krühler, T., Fynbo, J. P. U., et al. 2009, ApJ, 693, 1610

Greiner, J., Krühler, T., Klose, S., et al. 2011, A\&A, 526, A30

Groot, P. J., Galama, T. J., van Paradijs, J., et al. 1998, ApJ, 493, L27

Guidorzi, C., Kobayashi, S., Perley, D. A., et al. 2011, MNRAS, in press [arXiv: 1105.1591]

Güver, T., \& Özel, F. 2009, MNRAS, 400, 2050

Hashimoto, T., Ohta, K., Aoki, K., et al. 2010, ApJ, 719, 378

Hjorth, J., Sollerman, J., Møller, P., et al. 2003, Nature, 423, 847

Hopkins, A. M. 2004, ApJ, 615, 209

Ilbert, O., Capak, P., Salvato, M., et al. 2009, ApJ, 690, 1236

Ilbert, O., Salvato, M., Le Floc'h, E., et al. 2010, ApJ, 709, 644

Immler, S., Beardmore, A. P., Cummings, J. R., et al. 2008, GCN, 8500

Jakobsson, P., Hjorth, J., Fynbo, J. P. U., et al. 2004, ApJ, 617, L21

Jaunsen, A. O., Rol, E., Watson, D. J., et al. 2008, ApJ, 681, 453

Jin, Z. P., Xu, D., Covino, S., et al. 2009, MNRAS, 400, 1829

Kajisawa, M., Ichikawa, T., Tanaka, I., et al. 2009, ApJ, 702, 1393

Kann, D. A., Klose, S., \& Zeh, A. 2006, ApJ, 641, 993

Kann, D. A., Klose, S., Zhang, B., et al. 2010, ApJ, 720, 1513

Kann, D. A., Klose, S., Zhang, B., et al. 2011, ApJ, 734, 96

Kennicutt, Jr., R. C. 1998, ARA\&A, 36, 189

Klose, S., Stecklum, B., Masetti, N., et al. 2000, ApJ, 545, 271

Klose, S., Henden, A. A., Greiner, J., et al. 2003, ApJ, 592, 1025

Kobulnicky, H. A., \& Kewley, L. J. 2004, ApJ, 617, 240

Kocevski, D., West, A. A., \& Modjaz, M. 2009, ApJ, 702, 377

Kouveliotou, C., Meegan, C. A., Fishman, G. J., et al. 1993, ApJ, 413, L101

Krühler, T., Küpcü Yoldaş, A., Greiner, J., et al. 2008, ApJ, 685, 376

Krühler, T., Greiner, J., McBreen, S., et al. 2009, ApJ, 697, 758

Krühler, T., Schady, P., Greiner, J., et al. 2011, A\&A, 526, A153

Kuin, N. P. M., \& Immler, S. 2008, GCN, 8523

Küpcü Yoldaş, A., Salvato, M., Greiner, J., et al. 2007, A\&A, 463, 893

Küpcü Yoldaş, A., Greiner, J., Klose, S., Krühler, T., \& Savaglio, S. 2010, A\&A, 515, L2

Landolt, A. U. 1992, AJ, 104, 340

Le Floc'h, E., Duc, P., Mirabel, I. F., et al. 2003, A\&A, 400, 499

Levan, A., Fruchter, A., Rhoads, J., et al. 2006, ApJ, 647, 471

Levesque, E. M., Kewley, L. J., Graham, J. F., \& Fruchter, A. S. 2010, ApJ, 712, L26

MacFadyen, A. I., \& Woosley, S. E. 1999, ApJ, 524, 262

Malesani, D., Hjorth, J., Fynbo, J. P. U., et al. 2009, in AIPC Ser. 1111, ed. G. Giobbi et al., 513

Mannucci, F., Cresci, G., Maiolino, R., Marconi, A., \& Gnerucci, A. 2010a, MNRAS, 408, 2115

Mannucci, F., Salvaterra, R., \& Campisi, M. A. 2011, MNRAS, 414, 1263

Marchesini, D., van Dokkum, P., Quadri, R., et al. 2007, ApJ, 656, 42

Marchesini, D., van Dokkum, P. G., Förster Schreiber, N. M., et al. 2009, ApJ, 701,1765

Markwardt, C. B., Barthelmy, S. D., Baumgartner, W. H., et al. 2008, GCN, 8507

Michałowski, M. J., Hjorth, J., Castro Cerón, J. M., \& Watson, D. 2008, ApJ, 672,817

Milvang-Jensen, B., Goldoni, P., Tanvir, N. R., et al. 2010, GCN, 10876 
T. Krühler et al.: The SEDs and host galaxies of the dustiest GRB afterglows

Mokiem, M. R, de Koter, A, Vink, J. S., et al. 2007, A\&A, 473, 603 Monet, D. G., Levine, S. E., Canzian, B., et al. 2003, AJ, 125, 984 Naito, H., Sako, T., Suzuki, D., et al. 2010, GCN, 10881

Nardini, M., Ghisellini, G., Ghirlanda, G., \& Celotti, A. 2010, MNRAS, 403, 1131

Nardini, M., Greiner, J., Krúhler, T., et al. 2011, A\&A, 531, A39

Noll, S., \& Pierini, D. 2005, A\&A, 444, 137

Noll, S., Pierini, D., Cimatti, A., et al. 2009, A\&A, 499, 69

Oates, S. R., Page, M. J., Schady, P., et al. 2011, MNRAS, 412, 561

Palmer, D., Barthelmy, S. D., Baumgartner, W., et al. 2008, GCN, 8068

Oates, S. R., \& Ukwatta, T. N. 2010, GCN, 10878

Panaitescu, A., Mészáros, P., Burrows, D., et al. 2006, MNRAS, 369, 2059

Pei, Y. C. 1992, ApJ, 395, 130

Perley, D. A., Cenko, S. B., Bloom, J. S., et al. 2009, AJ, 138, 1690

Perley, D. A., Bloom, J. S., \& Cenko, S. B. 2010a, in BAAJ, 42, AAS Meeting Abstracts, 405.09

Perley, D. A., Bloom, J. S., Klein, C. R., et al. 2010b, MNRAS, 406, 2473

Perley, D. A., Bloom, J. S., Cenko, S. B., et al. 2011a, in AAS Meeting Abstracts, 217, 108.02

Perley, D. A., Morgan, A. N., Updike, A., et al. 2011b, AJ, 141, 36

Perna, R., Lazzati, D., \& Fiore, F. 2003, ApJ, 585, 775

Pickles, A. J. 1998, PASP, 110, 863

Pierini, D., Gordon, K. D., Witt, A. N., \& Madsen, G. J. 2004, ApJ, 617, 1022

Pontzen, A., Deason, A., Governato, F., et al. 2010, MNRAS, 402, 1523

Poole, T. S., Breeveld, A. A., Page, M. J., et al. 2008, MNRAS, 383, 627

Pozzetti, L., Bolzonella, M., Lamareille, F., et al. 2007, A\&A, 474, 443

Predehl, P., \& Schmitt, J. H. M. M. 1995, A\&A, 293, 889

Prochaska, J. X., Chen, H., \& Bloom, J. S. 2006, ApJ, 648, 95

Prochaska, J. X., Sheffer, Y., Perley, D. A., et al. 2009, ApJ, 691, L27

Ramirez-Ruiz, E., Trentham, N., \& Blain, A. W. 2002, MNRAS, 329, 465

Rau, A., Savaglio, S., Krühler, T., et al. 2010, ApJ, 720, 862

Rol, E., Wijers, R. A. M. J., Kouveliotou, C., Kaper, L., \& Kaneko, Y. 2005, ApJ, 624,868

Roming, P. W. A., Kennedy, T. E., Mason, K. O., et al. 2005, Space Sci. Rev., 120,95

Rossi, A., Schulze, S., Klose, S., et al. 2011a, A\&A, 529, A142

Rossi, A. et al. 2011b, A\&A, submitted

Salvaterra, R., Della Valle, M., Campana, S., et al. 2009, Nature, 461, 1258

Sari, R., Piran, T., \& Narayan, R. 1998, ApJ, 497, L17
Savaglio, S., \& Fall, S. M. 2004, ApJ, 614, 293

Savaglio, S., Fall, S. M., \& Fiore, F. 2003, ApJ, 585, 638

Savaglio, S., Glazebrook, K., Le Borgne, D., et al. 2005, ApJ, 635, 260

Savaglio, S., Glazebrook, K., \& Le Borgne, D. 2009, ApJ, 691, 182

Schady, P., Mason, K. O., Page, M. J., et al. 2007, MNRAS, 377, 273

Schady, P., Page, M. J., Oates, S. R., et al. 2010, MNRAS, 401, 2773

Schady, P., Savaglio, S., Krühler, T., Greiner, J., \& Rau, A. 2011a, A\&A, 525, A 113

Schady, P., et al. 2011b, A\&A, submitted

Schlegel, D. J., Finkbeiner, D. P., \& Davis, M. 1998, ApJ, 500, 525

Silva, L., Granato, G. L., Bressan, A., \& Danese, L. 1998, ApJ, 509, 103

Skrutskie, M. F., Cutri, R. M., Stiening, R., et al. 2006, AJ, 131, 1163

Stamatikos, M., Barthelmy, S. D., Baumgartner, W., et al. 2008, GCN, 7852

Starling, R. L. C., Wijers, R. A. M. J., Wiersema, K., et al. 2007, ApJ, 661, 787

Stratta, G., Fiore, F., Antonelli, L. A., Piro, L., \& De Pasquale, M. 2004, ApJ, 608,846

Stratta, G., D'Elia, V., \& Ukwatta, T. N. 2010, GCN, 10877

Tanvir, N. R., Barnard, V. E., Blain, A. W., et al. 2004, MNRAS, 352, 1073

Tanvir, N. R., Levan, A. J., Rol, E., et al. 2008, MNRAS, 388, 1743

Tanvir, N. R., Fox, D. B., Levan, A. J., et al. 2009, Nature, 461, 1254

Tody, D. 1993, in Astronomical Data Analysis Software and Systems II, ed. R. J. Hanisch, R. J. V. Brissenden, \& J. Barnes, ASP Conf. Ser., 52, 173

Ukwatta, T. N., Barthelmy, S. D., Baumgartner, W. H., et al. 2010a, GCN, 10875

Ukwatta, T. N., Barthelmy, S. D., Baumgartner, W. H., et al. 2010b, GCN, 10870

Updike, A., Nicuesa, A., Nardini, M., Kruehler, T., \& Greiner, J. 2010, GCN, 10874

van der Horst, A. J., Kouveliotou, C., Gehrels, N., et al. 2009, ApJ, 699, 1087

Vreeswijk, P. M., Ledoux, C., Smette, A., et al. 2007, A\&A, 468, 83

Watson, D., Fynbo, J. P. U., Ledoux, C., et al. 2006, ApJ, 652, 1011

Watson, D., Hjorth, J., Fynbo, J. P. U., et al. 2007, ApJ, 660, L101

Watson, D., French, J., Christensen, L., et al. 2011, ApJ, submitted [arXiv: 1010.1783$]$

Waxman, E., \& Draine, B. T. 2000, ApJ, 537, 796

Willmer, C. N. A., Faber, S. M., Koo, D. C., et al. 2006, ApJ, 647, 853

Witt, A. N., \& Gordon, K. D. 1996, ApJ, 463, 681

Woosley, S. E. 1993, ApJ, 405, 273

Yoon, S., \& Langer, N. 2005, A\&A, 443, 643

Zafar, T., Watson, D., Fynbo, J. P. U., et al. 2011, A\&A, 521, A143

Zhang, B. 2007, Chin. J. Astron. Astrophys, 7, 1 